\title{
Seasonal Mesoscale and Submesoscale Eddy Variability along the North Pacific Subtropical Countercurrent
}

\author{
Bo QIU AND SHUIMING CHEN \\ Department of Oceanography, University of Hawai'i at Mānoa, Honolulu, Hawaii \\ PATRICE KLEIN \\ Laboratoire de Physique des Océans, Ifremer/CNRS/UBO/IRD, Plouzané, France
}

HIDEHARU SASAKI

Application Laboratory, Japan Agency for Marine-Earth Science and Technology, Yokohama, Japan

YOSHIKAZU SASAI

Research and Development Center for Global Change, Japan Agency for Marine-Earth Science and Technology, Yokohama, Japan

(Manuscript received 4 April 2014, in final form 4 August 2014)

\begin{abstract}
Located at the center of the western North Pacific Subtropical Gyre, the Subtropical Countercurrent (STCC) is not only abundant in mesoscale eddies, but also exhibits prominent submesoscale eddy features. Output from a $1 / 30^{\circ}$ high-resolution OGCM simulation and a gridded satellite altimetry product are analyzed to contrast the seasonal STCC variability in the mesoscale versus submesoscale ranges. Resolving the eddy scales of $>150 \mathrm{~km}$, the altimetry product reveals that the STCC eddy kinetic energy and rms vorticity have a seasonal maximum in May and April, respectively, a weak positive vorticity skewness without seasonal dependence, and an inverse (forward) kinetic energy cascade for wavelengths larger (shorter) than $250 \mathrm{~km}$. In contrast, the submesoscale-resolving OGCM simulation detects that the STCC eddy kinetic energy and rms vorticity both appear in March, a large positive vorticity skewness with strong seasonality, and an intense inverse kinetic energy cascade whose short-wave cutoff migrates seasonally between the 35- and 100-km wavelengths. Using a 2.5-layer, reduced-gravity model with an embedded surface density gradient, the authors show that these differences are due to the seasonal evolution of two concurring baroclinic instabilities. Extracting its energy from the surface density gradient, the frontal instability has a growth time scale of $O(7)$ days, a dominant wavelength of $O(50) \mathrm{km}$, and is responsible for the surface-intensified submesoscale eddy signals. The interior baroclinic instability, on the other hand, extracts energy from the vertically sheared STCC system. It has a slow growth time scale of $O(40)$ days, a dominant wavelength of $O(250) \mathrm{km}$, and, together with the kinetic energy cascaded upscale from the submesoscales, determines the mesoscale eddy modulations.
\end{abstract}

\section{Introduction}

Accumulation of high-precision sea surface height (SSH) measurements from satellite altimeters in the past two decades has significantly improved our ability both to monitor the global ocean circulation variability

Corresponding author address: Dr. Bo Qiu, Department of Oceanography, University of Hawai'i at Mānoa, 1000 Pope Road, Honolulu, HI 96822.

E-mail: bo@soest.hawaii.edu and to explore its underlying dynamics. This improvement is particularly true with regard to our understanding of the oceanic mesoscale eddy signals that have temporal and spatial scales of 50-200 days and $100-500 \mathrm{~km}$, respectively. By taking advantage of concurrent altimeter missions, past studies have examined various aspects of the mesoscale eddies, ranging from their changes on multiple temporal-spatial scales, their generation and propagation, and their interaction with the background mean circulation to their impact upon heat, salt, and biogeochemical tracer transports. For comprehensive 


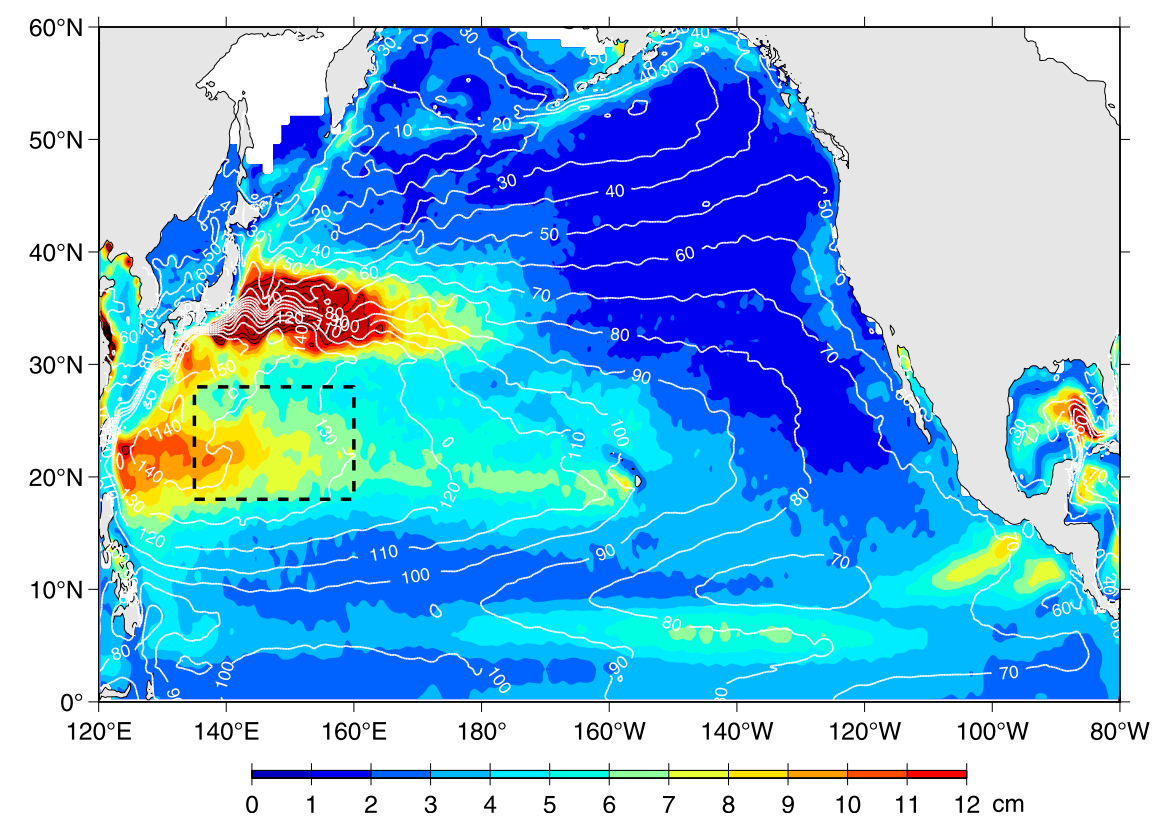

FIG. 1. Root-mean-square sea surface height variability in the North Pacific based on highpass filtered satellite altimeter data from October 1992 to February 2014. The high-pass filter has a half power at 180 days. Regions where the rms SSH variability exceeds $12 \mathrm{~cm}$ are indicated by thin black contours (with a contour interval at $2 \mathrm{~cm}$ ). White contours denote the mean sea surface height $(\mathrm{cm})$ field by Rio et al. (2011). Dashed box shows the STCC band of analyses.

reviews on the altimetry-based studies of the mesoscale eddies, readers are referred to $\mathrm{Fu}$ et al. (2010) and Morrow and Le Traon (2012).

In the North Pacific Ocean, one conspicuous band of high mesoscale eddy variability is located in the western half of the wind-driven subtropical gyre between $18^{\circ}$ and $28^{\circ} \mathrm{N}$ (Fig. 1). Although the wind-driven Sverdrup theory predicts a westward interior flow within this band, hydrographic observations reveal that the surface layer of this band is, in fact, occupied by a shallow eastward current (Fig. 2a). Known as the North Pacific Subtropical Countercurrent (STCC), this surface ocean eastward current has a mean speed of a few centimeters per second, and its formation as a time-mean current is due to the combined forcing of surface wind stress and heat fluxes [see the review by Kobashi and Kubokawa (2012) and the references therein]. The presence of the eastward STCC results in a positive meridional potential vorticity (PV) gradient in the upper 100-m ocean. Below this upper layer exists the wind-driven westward North Equatorial Current (NEC; see Fig. 2a). With the permanent thermocline associated with the NEC deepening toward north, the meridional PV gradient in the subsurface layer of $100 \mathrm{~m}$ to approximately $800 \mathrm{~m}$ is negative. This reversal in sign of the meridional PV gradient results in baroclinic instability and has been considered the energy source for the elevated eddy variability along the $18^{\circ}-28^{\circ} \mathrm{N}$ band in the western North Pacific Ocean (Qiu 1999; Roemmich and Gilson 2001; Kobashi and Kawamura 2002; Chang and Oey 2014).

An important aspect of the STCC eddy variability detected by the long-term satellite altimetry measurements is that the level of eddy kinetic energy (EKE) associated with the STCC has a well-defined annual cycle with a maximum in May and a minimum in December [see the red curve in Fig. 2b; as in many other studies of oceanic mesoscale eddy variability, we construct Fig. 2b using the SSH dataset compiled by Archiving Validation and Interpretation of Satellite Data in Oceanography (AVISO); see www.aviso.oceanobs. com]. Equally well defined is the annual cycle in rootmean-square (rms) vorticity within the STCC band as shown by the blue curve in Fig. $2 b$. The annual cycle in the EKE level has been argued to be due to the seasonal STCC changes that determine the growth of baroclinic instability. Specifically, the maximum growth, with an $e$-folding time scale of $O(2)$ months, occurs in March when the vertical shear of STCC-NEC is at maximum and the upper-ocean stratification is minimum (Qiu 1999; Kobashi and Kawamura 2002; Noh et al. 2007). The delay of the EKE maximum in May behind the instability peak in March has been interpreted as the time required for the initial perturbations of baroclinic instability to grow into finite-amplitude eddies. Instead of the exponential 


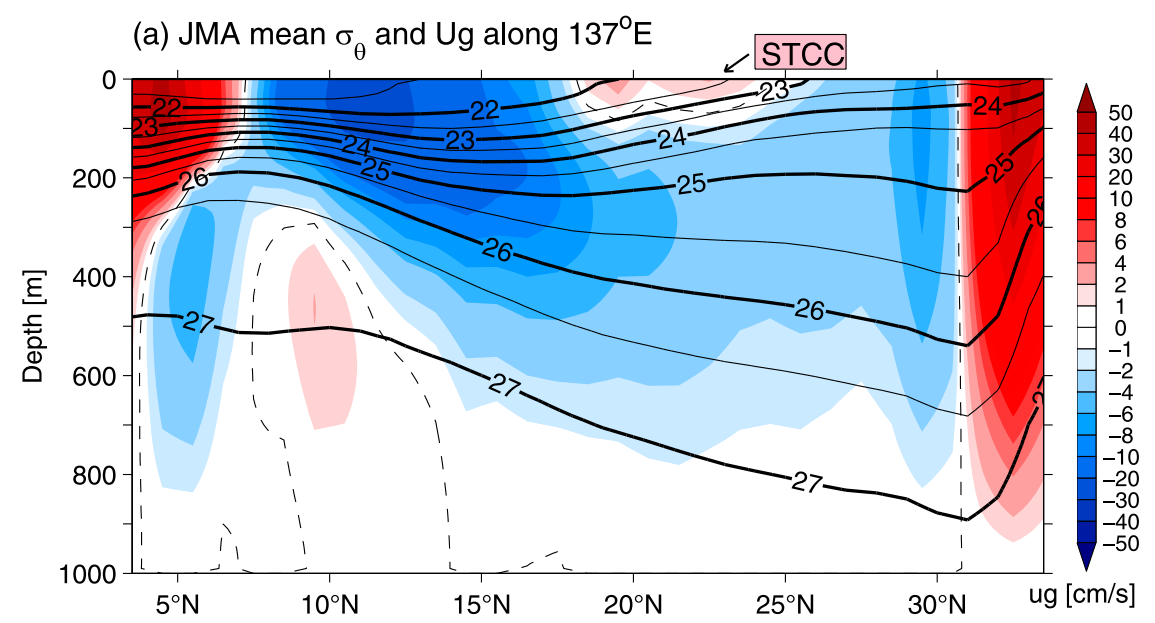

(b) AVISO EKE and $\zeta \mathrm{rms}$ in NP STCC

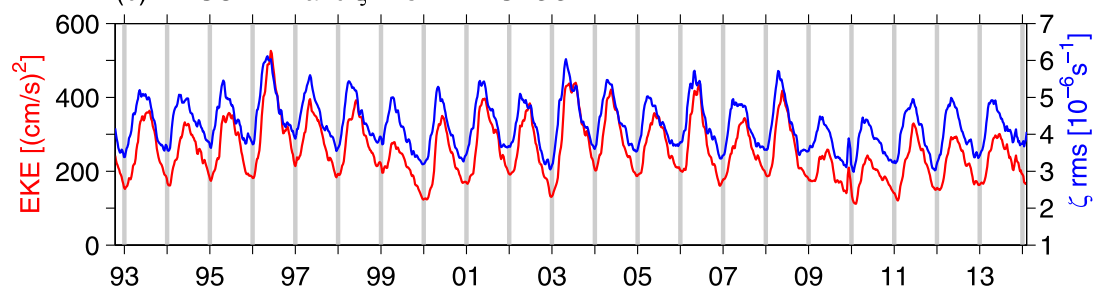

(c) AVISO Energy-Containing Length Scale
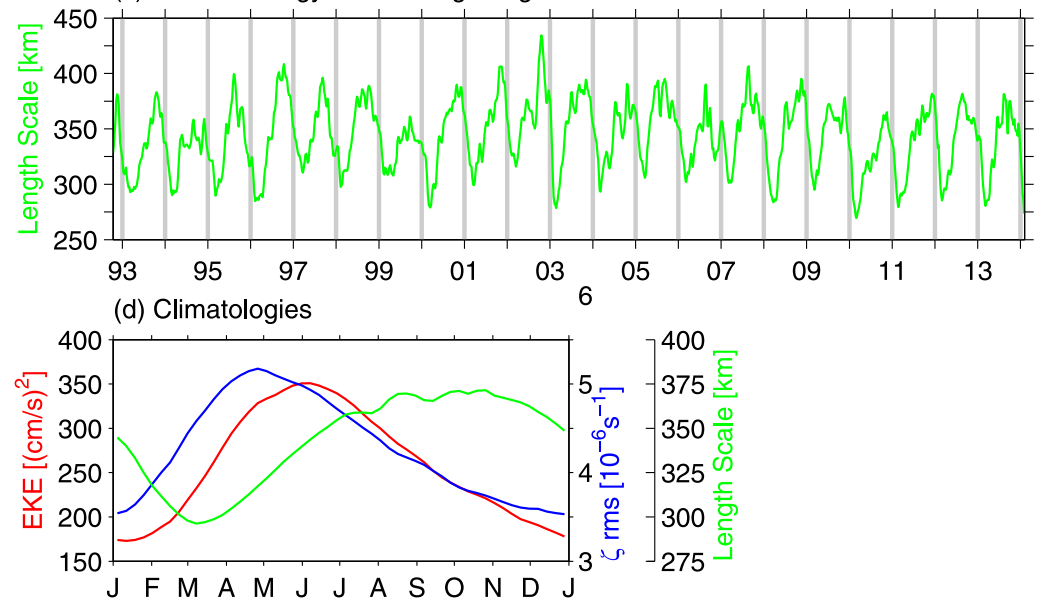

FIG. 2. (a) Latitude-depth section of temperature (solid contours) and zonal geostrophic velocity (color shading) along $137^{\circ} \mathrm{E}$ from the JMA repeat hydrographic surveys of $1993-2012$. The geostrophic velocity is referenced to $1000 \mathrm{dbar}$ and dashed lines denote the zero velocity contours. (b) EKE (red line) and rms relative vorticity (blue line) time series averaged in the STCC band of $18^{\circ}-28^{\circ} \mathrm{N}$ and $135^{\circ}-160^{\circ} \mathrm{E}$ based on the AVISO SSH data. (c) As in (b), but for the energy-containing length scale $L_{e}$ time series. (d) Annual cycle climatologies for EKE (red), rms relative vorticity (blue), and energy-containing length scale (green).

normal-mode growth, Chang and Oey (2014) have recently pointed to the possibility of a nonmodal growth in the STCC that has a faster $e$-folding time scale of $O(1)$ month.

It is worth mentioning that a lag correlation analysis reveals that the rms vorticity peaks in Fig. $2 b$ appear mostly in April, about 1 month earlier than the EKE peaks in May (the correlation coefficient is 0.95 when the rms vorticity time series leads the EKE time series by 1 month; see Fig. 2d). Dynamically, this 1-month lead by the rms vorticity signals was considered to be a consequence of the inverse cascade of kinetic energy (KE); as the STCCNEC becomes unstable, the initial eddy perturbations have a small length scale (limited by the resolution of AVISO here) that elevates the level of rms vorticity more 
effectively than the level of EKE (Qiu et al. 2008). As the eddy perturbations grow in amplitude, the inverse kinetic energy cascade leads to perturbations with broader length scales (see Fig. 2c), reducing the level of rms vorticity relative to that of EKE. Notice that the inverse kinetic energy cascade has been detected using altimeter data in the global oceans outside of the North Pacific STCC region as well (Scott and Wang 2005; Tulloch et al. 2011).

While our understanding of the seasonal STCC variability has advanced significantly due to the SSH information provided by the satellite altimeter missions, two areas remain to be clarified further. First, from an observational point of view, the spatial scales of the $\mathrm{SSH}$ signals resolvable by the multiple nadir-looking altimeters are longer than $O(150) \mathrm{km}$ (Chelton et al. 2011). With this limitation in spatial resolution, it is natural to ask if the shorter length scale eddy signals that are absent in the currently available SSH data product would alter the seasonal eddy characteristics of the STCC displayed, for example, in Figs. $2 b$ and 2c. This question is important and relevant because much of our current understanding of the seasonal STCC variability is rooted in the observed time series, such as those shown in Fig. 2.

Second, by adopting the quasigeostrophic (QG) potential vorticity dynamics, instability analysis studies in the past have focused on the seasonal vertical shear and stratification changes associated with the layered STCC-NEC system (Qiu 1999; Kobashi and Kawamura 2002). An inspection of available hydrographic surveys across the wintertime STCC reveals that a broad-scale, meridional density gradient exists within the surface 100-m layer (Fig. 3a). As indicated by the white curve in Fig. 3a, the 100-m depth corresponds roughly to the winter mixed layer depth in the region. In the presence of such an upper-ocean density gradient, previous theoretical and numerical modeling studies have indicated that instead of the QG potential vorticity dynamics, the emerging instability is governed by the ageostrophic frontal dynamics (e.g., Stone 1966; McCreary et al. 1991; Fukamachi et al. 1995; Spall 1995; Boccaletti et al. 2007; Klein et al. 2008; Capet et al. 2008b,c). While being a baroclinic instability that derives its energy from the mean potential energy of the background state, the frontal instability tends to have spatial eddy scales less than $100 \mathrm{~km}$ (i.e., the submesoscales) and to grow faster with a typical $e$-folding time scale of a few days. Notice that the existing studies of the frontal instability have often focused on coastal, or idealized oceanic, settings. In connection with the seasonal STCC variability of interest to this study, relevant questions include 1) does the frontal instability occur along the North Pacific STCC band; 2) if it does, how do its properties modulate with the season; and 3) to what extent does the frontal instability in the surface ocean contribute to the seasonally modulating mesoscale eddy signals?

To answer the questions listed above, we utilize in this study the output of a realistic, $1 / 30^{\circ}$-resolution, ocean general circulation model (OGCM) simulation of the North Pacific Ocean carried out at the Earth Simulator Center in the Japan Agency for Marine-Earth Science and Technology (JAMSTEC) (Sasaki and Klein 2012). After providing a detailed description of the model simulation and output in the next section, we examine in section 3 the eddy signals of various length scales in the high-resolution OGCM simulation and compare them to those detected by the satellite altimeter measurements. In section 4, we explore the dynamics governing the frontal instability and compare their properties with those of the interior baroclinic instability. Section 5 discusses how the concurring frontal and interior baroclinic instabilities control the seasonally modulating EKE, vorticity, and spectral kinetic energy fluxes in the STCC band, and section 6 summarizes the results from the present study.

\section{The $1 / 30^{\circ}$-resolution OFES simulation}

Since the early 2000s, the Earth Simulator Center of JAMSTEC has been conducting OGCM hindcast simulations of the global ocean at a mesoscale eddy-resolving resolution of $1 / 10^{\circ}$ in the horizontal and 54 levels in the vertical. The model is known as the OGCM for the Earth Simulator (OFES), and the model's numerical details and scientific results can be found in Masumoto et al. (2004), Sasaki et al. (2008), and the references cited therein. Recently, this mesoscale eddy-resolving OFES simulation was extended by Sasaki and Klein (2012) to a higher resolution of $1 / 30^{\circ}$ in the horizontal and 100 levels in the vertical, enabling it to capture many of the submesoscale eddy signals with wavelengths $\leq 100 \mathrm{~km}$ that were unresolved in the original $1 / 10^{\circ}$ OFES simulation.

As described in Sasaki and Klein (2012), the $1 / 30^{\circ}$ OFES simulation is performed within the North Pacific basin of $20^{\circ} \mathrm{S}-66^{\circ} \mathrm{N}$ and $100^{\circ} \mathrm{E}-70^{\circ} \mathrm{W}$. It uses the $1 / 10^{\circ}$ simulation output of 1 January 2000 as its initial condition and is forced by the 6-hourly surface wind stress and heat flux data of the Japanese 25-yr Reanalysis Project (JRA-25) product (Onogi et al. 2007). A biharmonic operator is used in the model for horizontal mixing of momentum and tracers, and Noh and Kim's (1999) scheme is adopted for the vertical mixing. The $1 / 30^{\circ}$ simulation has been integrated for $3 \mathrm{yr}$ and the second year (i.e., 2001) results will be used in this study for analyses. Notice that the simulation results of this year were analyzed by Sasaki and Klein (2012) with a focus on the geographical variation of SSH wavenumber spectra in the North Pacific Ocean. Using the $1 / 30^{\circ}$ OFES 
(a) JMA 137E $\sigma_{\theta}$ on Jan. 22-28, 2001

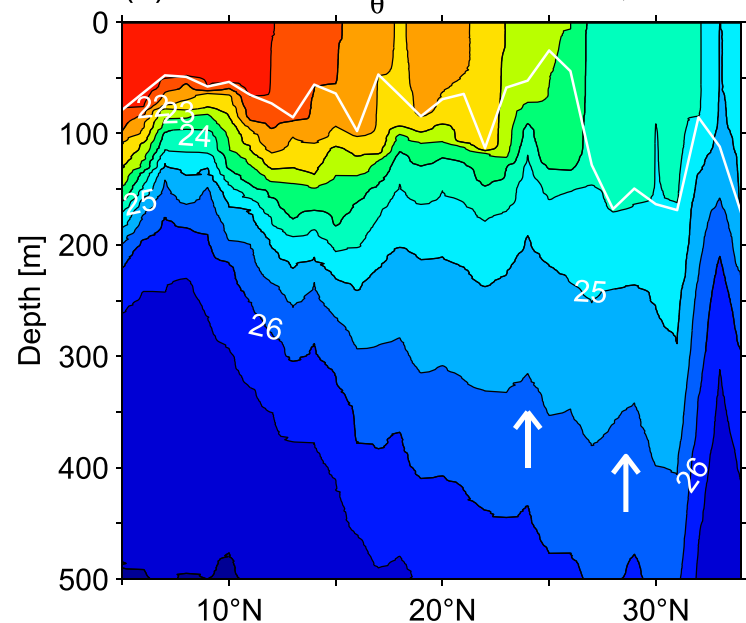

(c) OFES 137E $\sigma_{\theta}$ on Jan. 25, 2001

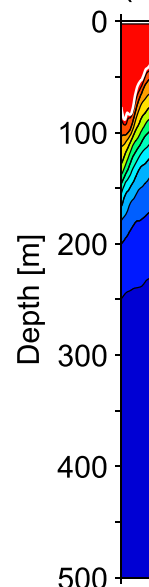

(b) JMA 137E $\sigma_{\theta}$ on Jul. 14-21, 2001

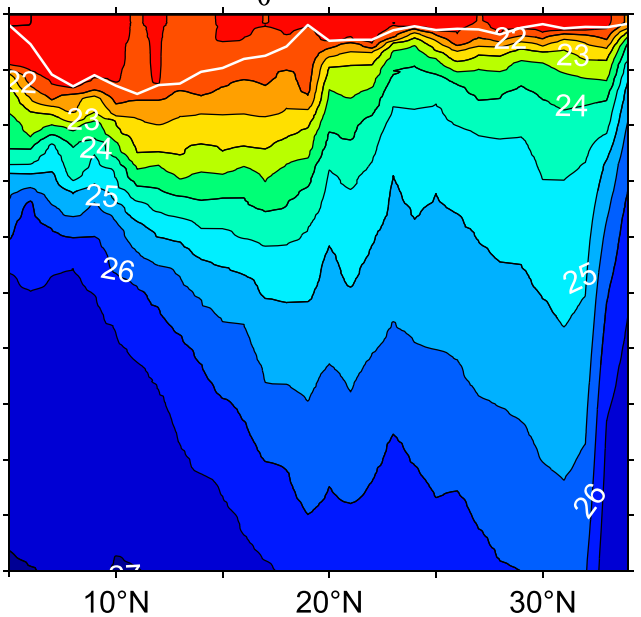

(d) OFES 137E $\sigma_{\theta}$ on Jul. 17, 2001

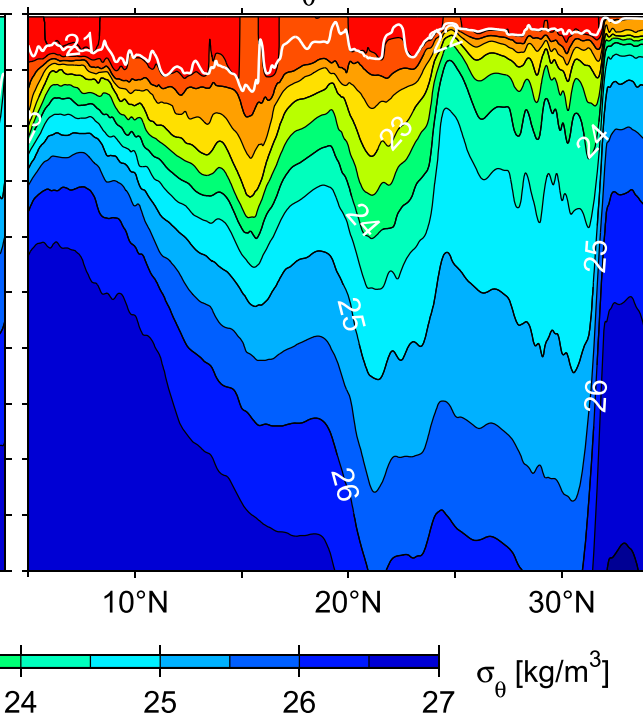

FIG. 3. (a) Latitude-depth section of density (color shading) and mixed layer depth (white line) along $137^{\circ} \mathrm{E}$ from the 2001 JMA hydrographic survey of 22-28 Jan. (b) As in (a), but for the survey of 14-21 Jul. (c) As in (a), but from the OFES simulation of $25 \mathrm{Jan}$. (d) As in (c), but for $17 \mathrm{Jul}$. White arrows in (a) and (c) denote the mesoscale "domings" noted in the text.

simulation output, Sasaki et al. (2014, manuscript submitted to Nat. Commun.) have more recently investigated seasonality of the submesoscale eddy activity in the Kuroshio Extension region. Readers interested in the basin-scale SSH and the submesoscale Kuroshio Extension variability are referred to these two publications.

\section{Simulated mesoscale versus submesoscale variability}

Before exploring the mesoscale and submesoscale variability in the STCC band in the $1 / 30^{\circ}$ OFES simulation, it is important to assess the extent to which the dynamical state of the seasonally varying STCC is adequately simulated by the OFES model. To do so, we plot in Fig. 3c the simulated density profile along $137^{\circ} \mathrm{E}$ on 25 January 2001. This date is chosen so that the simulated $\sigma_{\theta}(y, z)$ profile can be compared directly with the observed profile shown in Fig. 3a. The OFES simulation captures well the overall observed density structures, including the thickness and the poleward deepening of the mixed layer due to the increased heat loss toward the wintertime higher latitudes (Qiu et al. 2004). Despite the turbulent nature of the ocean, both the model and observations exhibit mesoscale isopycnal domings at the latitudes near $23^{\circ}$ and $28^{\circ} \mathrm{N}$ below the mixed layer (see white arrows in Figs. $3 a$ and $3 c$ ). 
(a) OFES $\sigma_{\theta}: 10 \mathrm{~m}, 135-160^{\circ} \mathrm{E}$

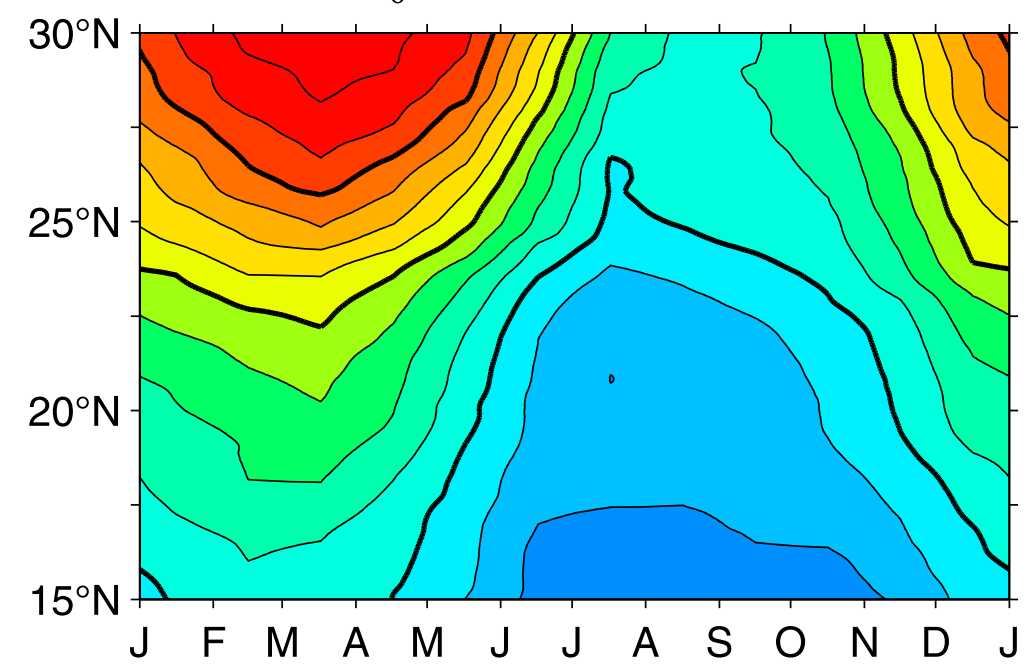

(b) MOAA $\sigma_{\theta}: 10 \mathrm{~m}, 135-160^{\circ} \mathrm{E}$

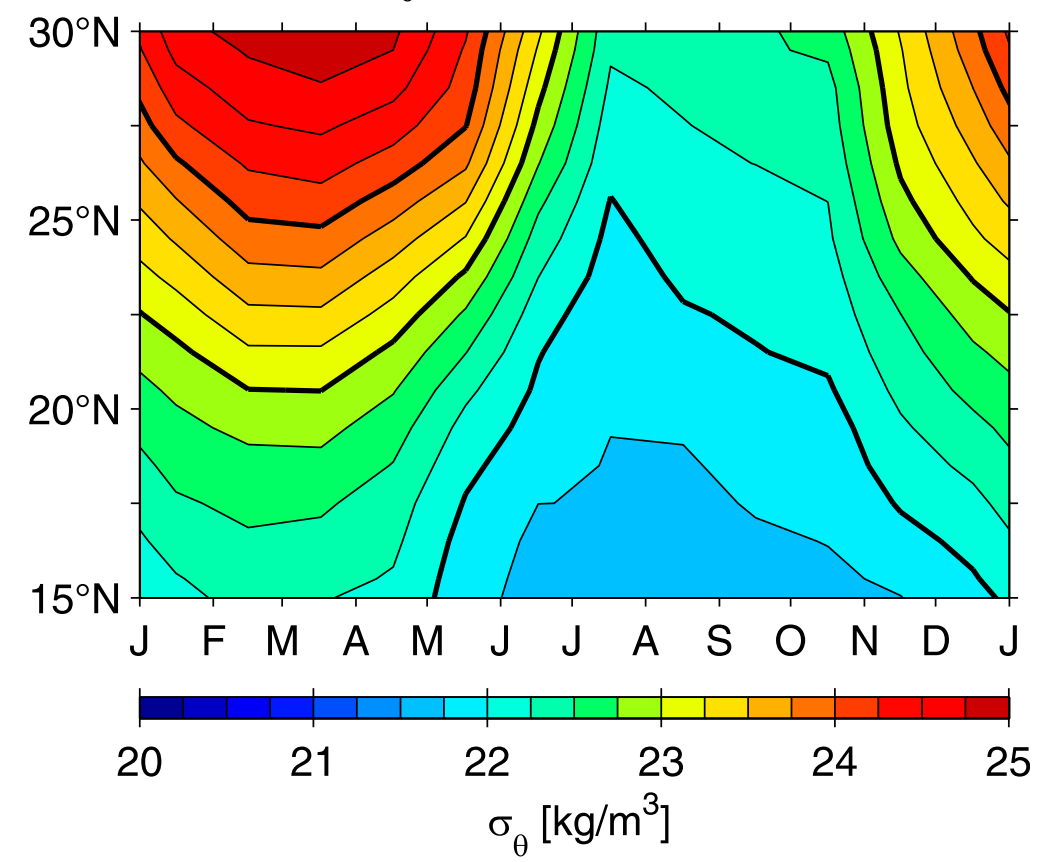

FIG. 4. Zonally averaged $\left(135^{\circ}-160^{\circ} \mathrm{E}\right)$ density distribution at $10-\mathrm{m}$ depth as a function of time and latitude based on (a) the OFES simulation of 2001 and (b) the objectively mapped temperature dataset of Hosoda et al. (2008).

This favorable comparison is not limited to the winter case. Figures $3 \mathrm{~b}$ and $3 \mathrm{~d}$ show the observed and simulated density profiles in mid-July along $137^{\circ} \mathrm{E}$; the shoaling of the mixed layer and the increased stratification within the upper $100-\mathrm{m}$ layer north of $18^{\circ} \mathrm{N}$ are discernible in both the observations and the OFES simulation.

Given the importance of the meridional density gradient in the upper ocean for the frontal instability, we compare in Fig. 4 the observed and modeled density values at the $10-\mathrm{m}$ depth zonally averaged from $135^{\circ}$ to $165^{\circ} \mathrm{E}$ as a function of time and latitude. Here, the observed density is based on the 2001 monthly temperaturesalinity dataset compiled by Hosoda et al. (2008) from the global Argo float and other hydrographic measurements, and the zonal average is taken in order to emphasize the coherent density changes associated with the 
(a) EKE at Surface

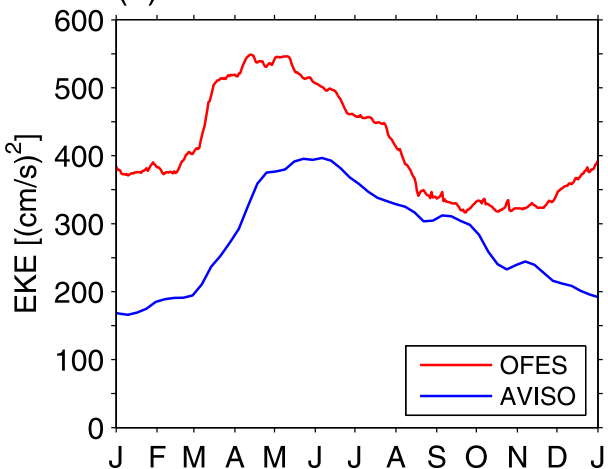

(b) EKE at Surface: MS vs. SM

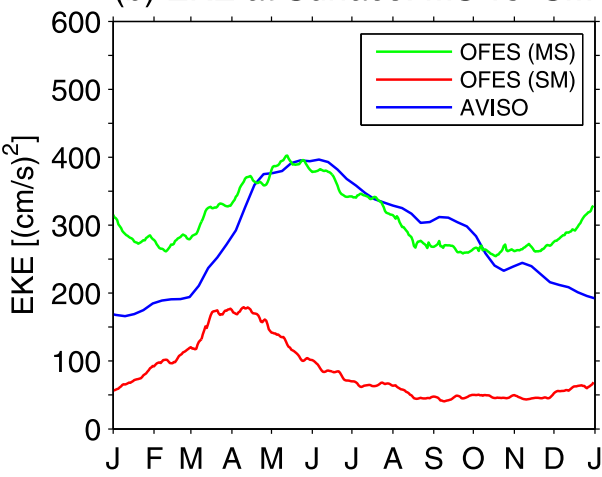

(c) $\zeta$ rms at Surface

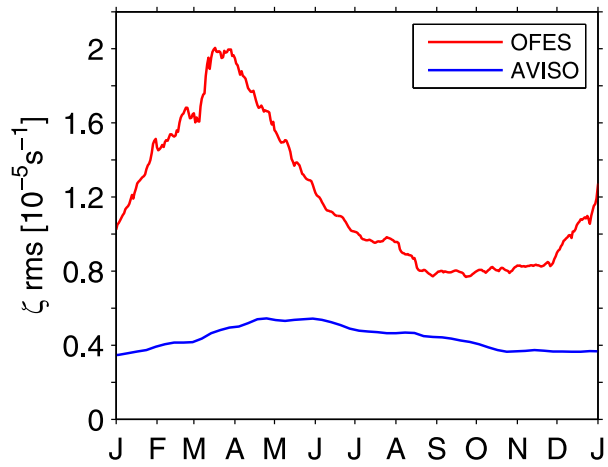

(d) EKE (MS)

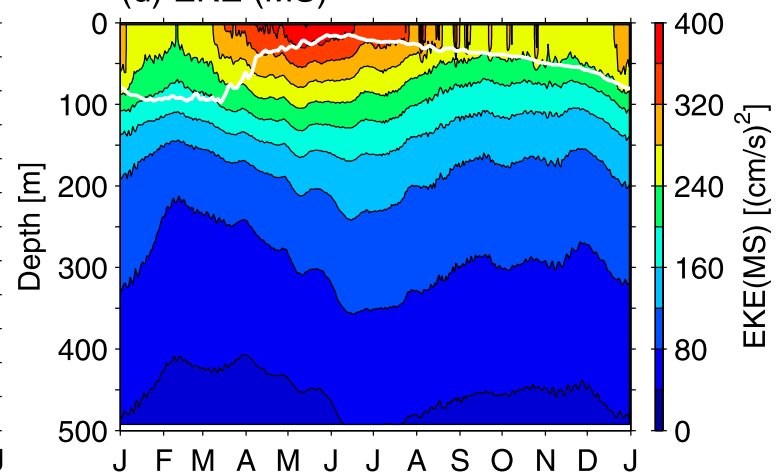

(e) EKE (SM)

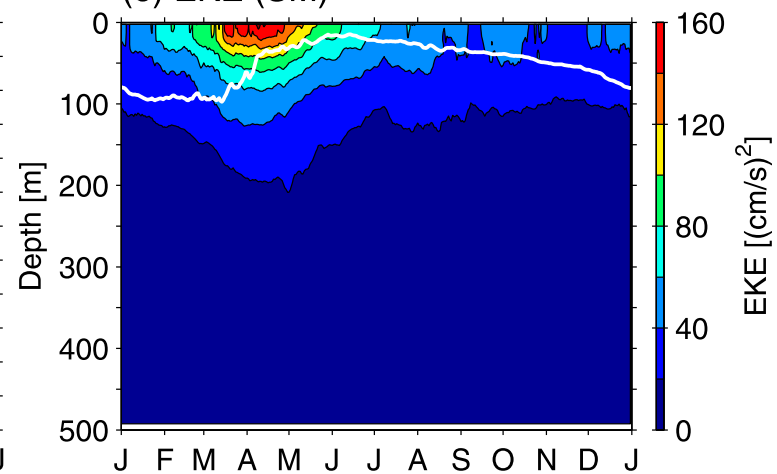

(f) $\zeta \mathrm{rms}$ (OFES)

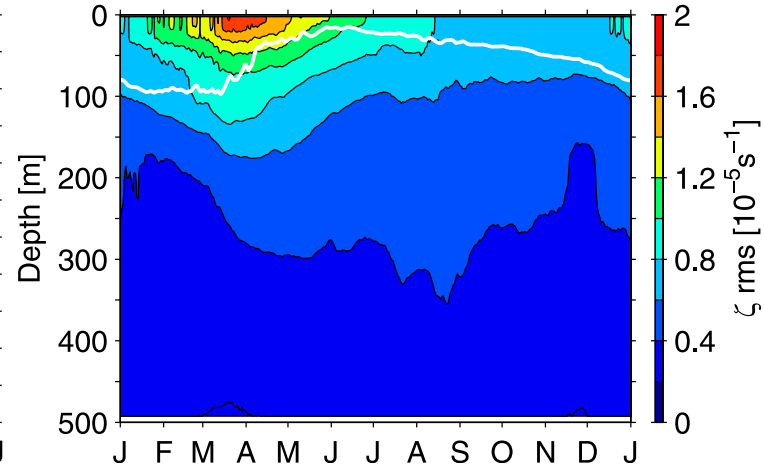

FIG. 5. (a) Surface EKE time series from the OFES simulation (red line) vs the AVISO SSH data (blue line) in 2001. (b) Time series of mesoscale EKE (green line) vs submesoscale EKE (red line) from the OFES simulation. Blue line is same as that in (a). (c) As in (a), but for the surface relative vorticity time series. Vertical distributions of (d) mesoscale EKE, (e) submesoscale EKE, and (f) relative vorticity from the OFES simulation. White lines denote the mixed layer depth.

seasonally evolving STCC. Similar to the case of vertical density profiles presented in Fig. 3, the OFES model simulates well the seasonal evolution of the upper-ocean density field. Notice that the meridional density gradient across the STCC reaches a maximum in March and a minimum in September.

Following the analysis of satellite altimeter data, we plot in Fig. 5a (red line) the EKE time series in the STCC band $\left(18^{\circ}-28^{\circ} \mathrm{N}, 135^{\circ}-160^{\circ} \mathrm{E}\right)$ from the OFES simulation:

$$
\mathrm{EKE}=\frac{g^{2}}{2 f^{2}}\left[\left(\frac{\partial \eta^{\prime}}{\partial x}\right)^{2}+\left(\frac{\partial \eta^{\prime}}{\partial y}\right)^{2}\right]
$$

where $\eta^{\prime}$ is the SSH anomaly, $g$ is the gravity constant, and $f$ is the Coriolis parameter. For comparison, we superimpose in Fig. 5a the EKE time series calculated from the 2001 AVISO SSH anomaly data (blue line). There are two noticeable differences. First, the mean 
EKE level in the OFES simulation is $47 \%$ higher than that of the AVISO result. This difference in the EKE level is not probably surprising given that the AVISO product only resolves the eddy signals with length scales greater than $O(150) \mathrm{km}$. A second surprising difference in Fig. 5a is that the EKE peak in the OFES simulation occurs in April, $\sim 1.5$ months earlier than the AVISO result.

To understand this second difference, we divide the OFES $\eta^{\prime}$ signal into

$$
\eta^{\prime}(x, y, t)=\eta_{\mathrm{MS}}^{\prime}(x, y, t)+\eta_{\mathrm{SM}}^{\prime}(x, y, t),
$$

where $\eta_{\mathrm{MS}}^{\prime}$ and $\eta_{\mathrm{SM}}^{\prime}$ denote the $\eta^{\prime}$ signals with wavelengths longer (MS) and shorter (SM) than $150 \mathrm{~km}$, respectively. For brevity, we will hereafter refer to the eddy signals with length scales longer (shorter) than $150 \mathrm{~km}$ as mesoscale (submesoscale). The green line in Fig. 5b shows the EKE time series calculated from Eq. (1) using the mesoscale SSH data $\eta_{\mathrm{MS}}^{\prime}$. This mesoscale EKE time series is in reasonable agreement with the AVISOderived EKE time series (blue line) in terms of the mean energy level and the timing of seasonal energy peak, confirming that the currently available AVISO SSH product is capable of capturing the mesoscale eddy signals with length scales exceeding $150 \mathrm{~km}$ only. The red line in Fig. 5b shows the EKE time series calculated based on the OFES submesoscale $\eta_{\mathrm{SM}}^{\prime}$ signals. In contrast to the mesoscale EKE, elevated submesoscale EKE is largely confined to February-May. Notice that the submesoscale EKE peaks between mid-March and mid-April, the period in which the upper ocean has a seasonally maximum meridional density gradient (recall Fig. 4a).

Unlike the satellite altimeter data, the OFES simulation also provides us with the EKE signals below the sea surface. Figures $5 \mathrm{~d}$ and $5 \mathrm{e}$ compare the simulated mesoscale and submesoscale EKE signals as a function of time and depth. For the mesoscale EKE signals, Fig. 5d reveals that in addition to the decrease in energy level with increasing depth, the seasonal EKE peak at deep levels lags the surface peak by about a month (June versus May). In contrast to the mesoscale EKE, Fig. 5e reveals that the submesoscale EKE is more surface trapped and has a vertically coherent seasonal peak in late March and early April. In fact, a significant part of the submesoscale EKE is confined within the surface mixed layer, whose depth is indicated in Fig. $5 \mathrm{~d}$ by a white line. An exception to this occurs in early spring when some of the submesoscale EKE are left behind beneath the rapidly shoaled mixed layer. As the season progresses, the submesoscale EKE signals both within and below the mixed layer rapidly dissipate.

Compared to the EKE signals, the difference between the simulated and AVISO-derived surface relative vorticity signals is more dramatic. As shown in Fig. 5c, the simulated rms vorticity time series has a much more prominent annual cycle than that inferred geostrophically from the AVISO SSH data (blue line). In the introduction, we noted that the AVISO-derived rms vorticity maximum leads the EKE maximum by a month (recall Fig. 2d). Interestingly, this 1-month lead is also seen between the simulated total EKE and rms vorticity maxima (cf. the red lines in Figs. 5a and 5c). While leading the total EKE by 1 month, the rms surface vorticity exhibits an in phase annual cycle similar to that of the submesoscale EKE time series (i.e., the solid red line in Fig. 5b), reconfirming that relative vorticity is controlled preferentially by small-scale perturbations. Vertically, the rms vorticity distribution (Fig. 5f) shows a pattern in between Figs. $5 \mathrm{~d}$ and $5 \mathrm{e}$; it is surface trapped in winter like the submesoscale EKE pattern, but extends to deeper ocean in the summer and fall seasons due to the influence from deep-reaching mesoscale eddies.

It is worth noting that not only does the amplitude of the simulated rms vorticity fluctuate between different seasons, the dynamical characteristics of vorticity are also different depending on the season. To illustrate this point visually, we contrast in Fig. 6 the simulated surface vorticity snapshots from 1 March versus 1 September in the western North Pacific Ocean. Within the STCC box of interest to this study, there exists a clear difference in spatial scales of the omnipresent eddies and filaments. To quantify the seasonal evolution of the spatial eddy scales, we plot in Fig. 7a (red line) the time series of energy-containing length scale $L_{e}$ in the STCC box from the OFES simulation. Here, the energy-containing length scale is defined by

$$
L_{e}=\frac{2 \pi \iint \tilde{E}(k, l) d k d l}{\iint \sqrt{k^{2}+l^{2}} \tilde{E}(k, l) d k d l},
$$

where $\tilde{E}(k, l)$ denotes the power spectral density of EKE, and $k$ and $l$ are the zonal and meridional wavenumbers, respectively. The minimum eddy scale $L_{e} \simeq 165 \mathrm{~km}$ is obtained in March when the submesoscale EKE and rms relative vorticity are both at their seasonal maxima. From April to November, Fig. 7a reveals that $L_{e}$ has a generally increasing trend, and this trend has a larger rate during April-June than during July-November. The average $L_{e}$ value during July-November is $\sim 290 \mathrm{~km}$, a $75 \%$ increase over the March $L_{e}$ value. With the regional first baroclinic Rossby radius of deformation $L_{R}$ estimated at $\sim 50 \mathrm{~km}$ (Chelton et al. 1998), the simulated $L_{e}$ is much smaller than $2 \pi L_{R}$ in March, whereas it is close to $2 \pi L_{R}$ during the summer and fall seasons. 
(a) $\zeta$ field: 03/01/2001

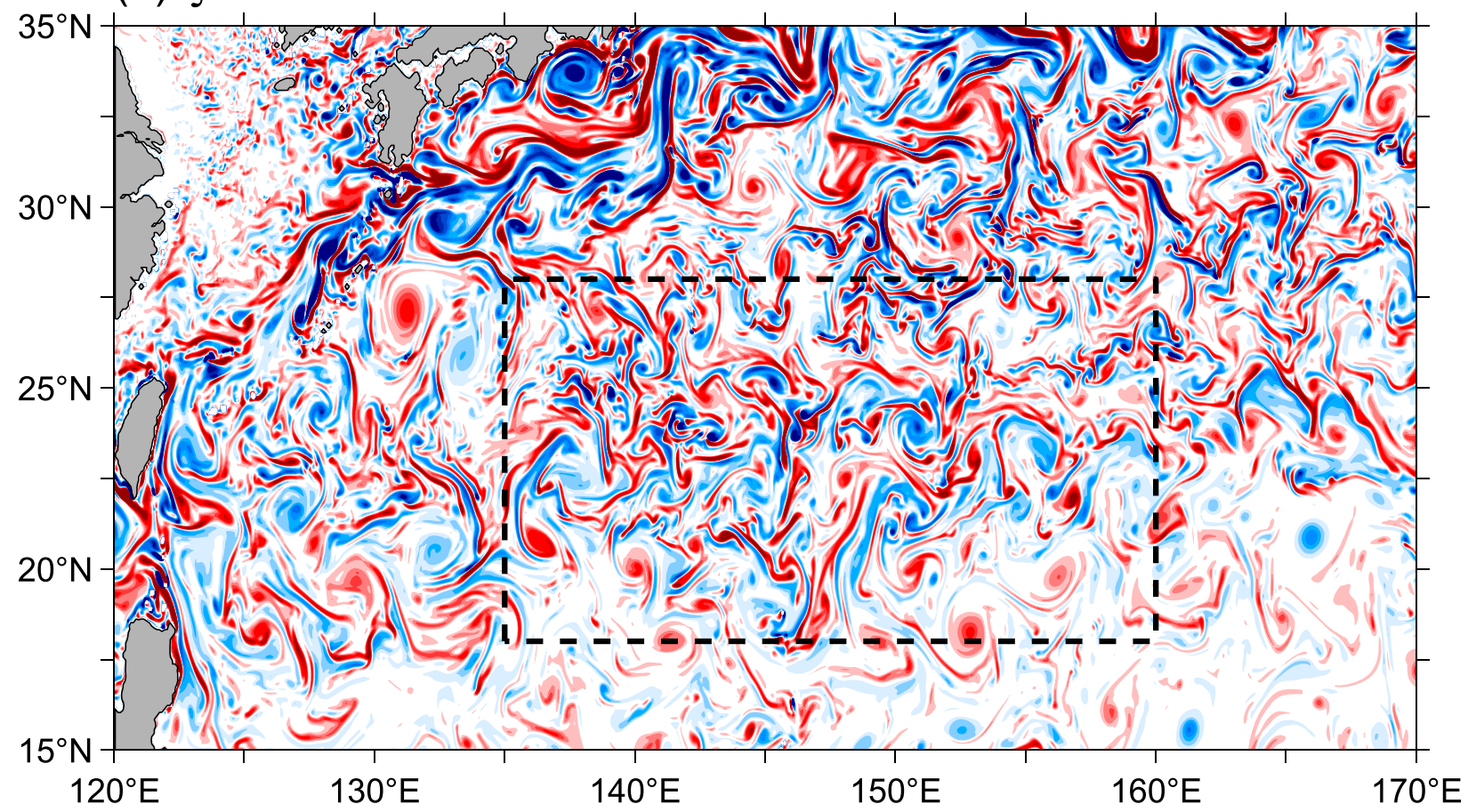

(b) $\zeta$ field: 09/01/2001

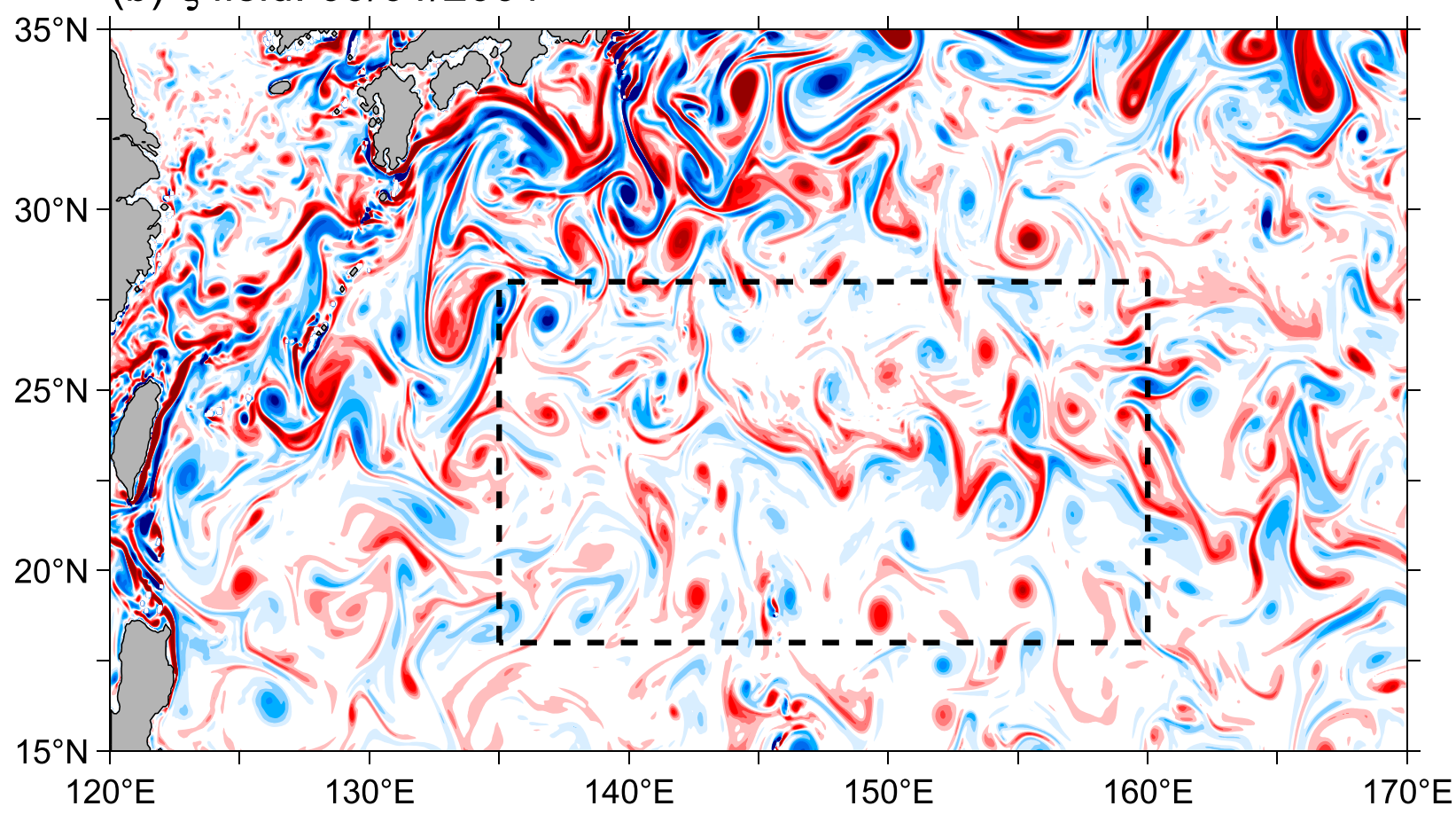

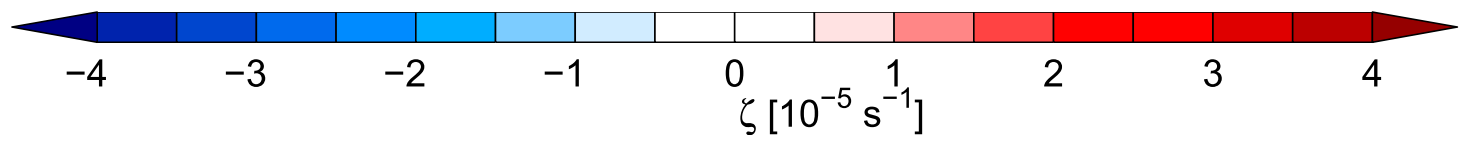

FIG. 6. Snapshots of surface relative vorticity in the western North Pacific Ocean from the OFES simulation: (a) 1 Mar and (b) 1 Sep 2001. Dashed box indicates the STCC region analyzed in this study. 
(a) Energy-Containing Length Scale

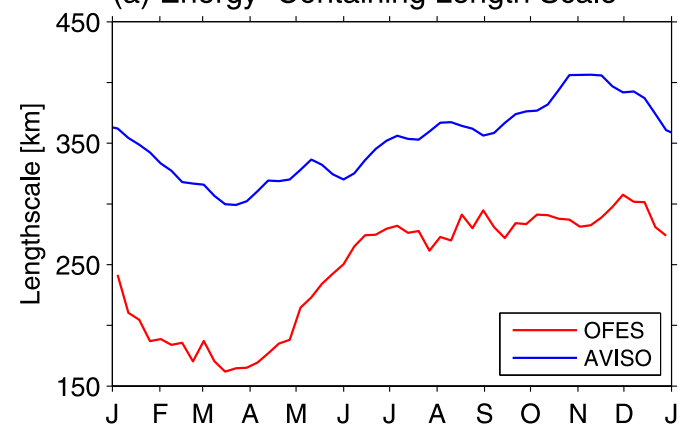

(b) OFES $\zeta / f$ PDF

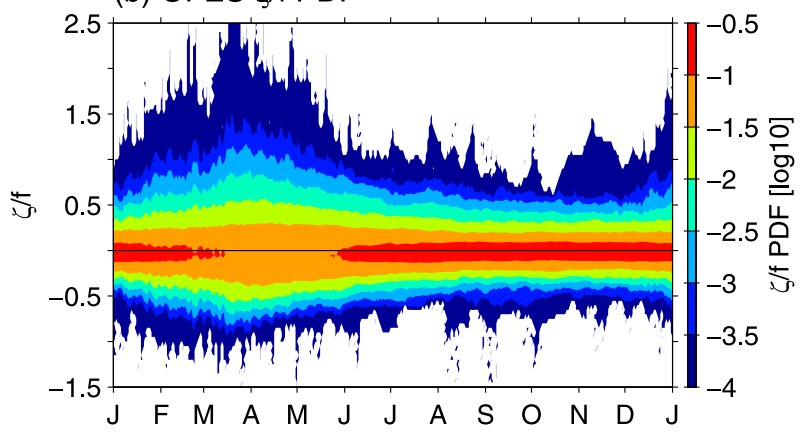

(c) $\zeta / f$ Skewness

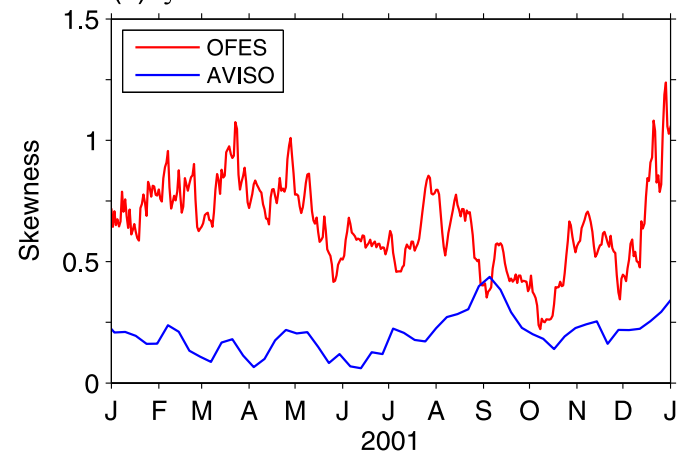

FIG. 7. (a) Energy-containing length scale $L_{e}$ time series from the OFES simulation (red line) vs the AVISO SSH data (blue line) in 2001. (b) Time series of normalized relative vorticity $(\zeta / f)$ pdf from the OFES simulation. (c) As in (a), but for the time series of $\zeta / f$ skewness.

A second, and less visually obvious, difference between the relative vorticity signals presented in Fig. 6 is that compared to September; the positive vorticity features in March are more predominant than the negative vorticity features. This seasonally varying asymmetry in relative vorticity can be quantified by examining the probability density function (pdf) of relative vorticity normalized by the local Coriolis parameter $f($ i.e., $\zeta / f$ ) as a function of time. As shown in Fig. 7b, whereas the magnitudes of negative $\zeta$ rarely exceed $f$, the positive vorticity amplitudes are frequently above $f$ in the winterspring months from December to May. Notice that the lower cutoff near $\zeta / f<-1$ is indicative of centrifugal instability limitations.
A different way of quantifying this vorticity asymmetry is to calculate the skewness of the normalized relative vorticity (the red line in Fig. 7c):

$$
S=\frac{1}{N} \sum_{n=1}^{N}(\zeta \mid f-\langle\zeta \mid f\rangle)^{3} /\left[\frac{1}{N} \sum_{n=1}^{N}(\zeta / f-\langle\zeta \mid f\rangle)^{2}\right]^{2 / 3},
$$

where $N$ denotes the number of grid points, and \langle\rangle is the spatial averaging in the STCC box. In accordance with the pdf result shown in Fig. 7b, the relative vorticity is skewed positively, reflecting the predominance of cyclonic features over the anticyclonic ones. In particular, large positive skewness values are detected in the winterspring months when the level of rms vorticity is seasonally high. Existence of increased skewness in the submesoscale range has been detected previously in in situ observations and high-resolution ocean simulations (e.g., Munk et al. 2000; Rudnick 2001; Eldevik and Dysthe 2002; Boccaletti et al. 2007; Klein et al. 2008). The result of Fig. 7c suggests such increased skewness is seasonally modulated.

Before exploring the dynamical processes responsible for the seasonal length scale and vorticity modulations, it is of interest to compare the OFES simulation results presented in Fig. 7 with those captured by the AVISO satellite altimeter data. To do so, we superimpose in Fig. 7a (blue line) the $L_{e}$ values estimated using Eq. (3) based on the 2001 AVISO SSH data. By and large, the AVISO-derived $L_{e}$ time series shows a seasonal modulation similar to that detected in the OFES simulation. By failing to capture the eddy signals with length scales shorter than $150 \mathrm{~km}$, however, the mean $L_{e}$ value inferred from the AVISO data is overestimated by $\sim 100 \mathrm{~km}$. In terms of the skewness for normalized relative vorticity, the blue line in Fig. 7c indicates that while capturing a positive-valued skewness, the AVISO-inferred time series completely misses the seasonal $\zeta / f$ skewness modulation seen in the OFES simulation. This miss is not surprising, given that the seasonally modulating vorticity asymmetry is caused by wintertime emergence of finescale vorticity signals that are absent in the AVISO SSH dataset.

\section{Frontal versus interior baroclinic instabilities}

The analyses of the $1 / 30^{\circ}$-resolution OFES simulation in the preceding section indicated the coexistence of mesoscale and submesoscale eddy signals along the North Pacific STCC band of $18^{\circ}-28^{\circ} \mathrm{N}$. Energetically, these eddy signals show subtly different seasonal modulations with the submesoscale EKE peaking in March and leading the mesoscale EKE peak by 2 months. With 


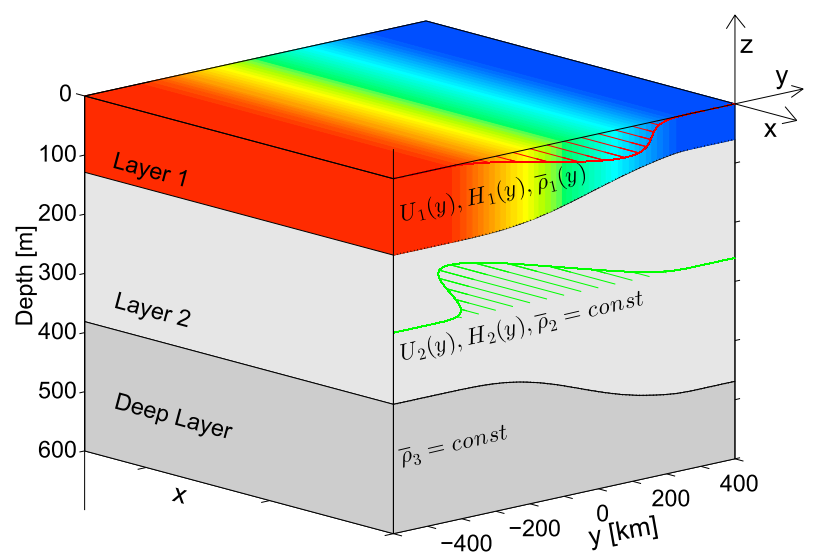

FIG. 8. Schematic of the 2.5-layer STCC-NEC reduced-gravity model that includes a $y$-dependent density variation (color shade) in the upper layer.

regard to the mesoscale EKE signals, their seasonal modulation in the OFES simulation is in accordance with the AVISO observations. As we reviewed in the introduction, baroclinic instability caused by the seasonally varying shear between the surface eastwardflowing STCC and the subsurface westward-flowing NEC has been proposed in the past to be responsible for the seasonal EKE modulation observed by AVISO (e.g., Qiu 1999; Kobashi and Kawamura 2002). This interior baroclinic instability mechanism, however, is not applicable to the submesoscale EKE signals prevalent during the winter season when the surface mixed layer is deep and accompanied by a large meridional density gradient (recall Fig. 4).

To examine how the presence of the meridional density gradient in the winter mixed layer impacts the instability characteristics of the vertically sheared STCC-NEC system, we extend in this section our previous 2.5 -layer, reduced-gravity model by allowing for the density gradient in the upper layer to change meridionally. Such a density-dependent, 2.5-layer model, as schematically illustrated in Fig. 8, has been utilized in the past by McCreary et al. (1991) in their investigation of the upper-ocean frontal instability near an eastern ocean boundary. In the context of this study, the upper layer in Fig. 8 represents the winter mixed layer in which the eastward-flowing STCC $U_{1}(y)$ exists and the $y$-dependent density $\bar{\rho}_{1}(y)$ is externally imposed. The subsurface lower layer is occupied by the westwardflowing NEC $U_{2}(y)$ and its density is fixed at constant $\bar{\rho}_{2}$. Layer three has a constant density $\bar{\rho}_{3}$ and is assumed to be infinitely deep and inert.

The linearized momentum, continuity, and upperlayer density equations governing the evolution of small-amplitude perturbations in the 2.5-layer, reducedgravity model are (see McCreary et al. 1991)

$$
\begin{gathered}
\frac{\partial u_{i}}{\partial t}+U_{i} \frac{\partial u_{i}}{\partial x}+v_{i} \frac{\partial U_{i}}{\partial y}-f v_{i}=-\frac{1}{\rho_{0}} \frac{\partial p_{i}}{\partial x}-\nu_{4} \nabla^{4} u_{i} \\
\frac{\partial v_{i}}{\partial t}+U_{i} \frac{\partial v_{i}}{\partial x}+f u_{i}=-\frac{1}{\rho_{0}} \frac{\partial p_{i}}{\partial y}-\nu_{4} \nabla^{4} v_{i} \\
\frac{\partial h_{i}}{\partial t}+U_{i} \frac{\partial h_{i}}{\partial x}+v_{i} \frac{\partial H_{i}}{\partial y}+H_{i}\left(\frac{\partial u_{i}}{\partial x}+\frac{\partial v_{i}}{\partial y}\right)=0, \quad \text { and } \\
\frac{\partial \rho_{1}}{\partial t}+U_{1} \frac{\partial \rho_{1}}{\partial x}+v_{1} \frac{\partial \bar{\rho}_{1}}{\partial y}=0
\end{gathered}
$$

where $\left(u_{i}, v_{i}\right)$ are the perturbation velocities in layer $i$ $(i=1,2), h_{i}$ is the $i$ th layer perturbation thickness, $H_{i}$ is the $i$ th layer mean thickness, $\rho_{1}$ is the upper-layer perturbation density, $\rho_{0}$ is the reference density, and $\nu_{4}$ is the biharmonic eddy viscosity coefficient. In Eqs. (5) and (6), the perturbation pressure gradient is given by

$$
\begin{aligned}
\nabla p_{1}= & -g \nabla\left(h_{1} \bar{\rho}_{13}+h_{2} \bar{\rho}_{23}+H_{1} \rho_{1}\right) \\
& +\frac{1}{2} g\left(h_{1} \nabla \bar{\rho}_{1}+H_{1} \nabla \rho_{1}\right), \\
\nabla p_{2}= & -g \bar{\rho}_{23} \nabla\left(h_{1}+h_{2}\right),
\end{aligned}
$$

where $\bar{\rho}_{i j}=\bar{\rho}_{i}-\bar{\rho}_{j}$, and the biharmonic eddy viscosity terms are included for direct comparison with the OFES simulation results (as in the OFES simulation, $\nu_{4}=$ $10^{-9} \mathrm{~m}^{4} \mathrm{~s}^{-1}$ is used). In the limit of $\nu_{4}=0$ and an absent density gradient in the upper layer, $\nabla p_{1}$ simplifies to $-g\left(\bar{\rho}_{13} \nabla h_{1}+\bar{\rho}_{23} \nabla h_{2}\right)$, and the governing Eqs. (5)-(8) may be combined to form the perturbation potential vorticity equations for the upper and lower layers [see Eq. (2) in Qiu 1999].

To explore how baroclinic instability is modified by the inclusion of $\bar{\rho}_{1}(y)$, we assume STCC and NEC in the upper and lower layers have the following, idealized, $y$-dependent profiles:

$$
U_{i}(y)= \begin{cases}\frac{1}{2} A_{i}\left[1+\cos \frac{2 \pi\left(y-y_{0}\right)}{L}\right], & \left|y-y_{0}\right| \leq L / 2 \\ 0, & \text { otherwise }\end{cases}
$$

where $A_{i}, y_{0}$, and $L$ denote the maximum speed, center latitude, and width of STCC and NEC, respectively. Based on Fig. 4, we further assume that the upper-ocean density has a linear meridional profile:

$$
\bar{\rho}_{1}(y)= \begin{cases}\bar{\rho}_{10}-\Delta \rho / 2, & y-y_{0}<-L / 2 \\ \bar{\rho}_{10}+\left(y-y_{0}\right) \Delta \rho / L, & \left|y-y_{0}\right| \leq L / 2 \\ \bar{\rho}_{10}+\Delta \rho / 2, & y-y_{0}>L / 2\end{cases}
$$


TABLE 1. Parameter values appropriate for the 2.5-layer STCC-NEC system in March.

\begin{tabular}{cc}
\hline \hline Parameter & Value \\
\hline$A_{1}$ & $3 \mathrm{~cm} \mathrm{~s}^{-1}$ \\
$A_{2}$ & $-3 \mathrm{~cm} \mathrm{~s}^{-1}$ \\
$H_{10}$ & $100 \mathrm{~m}$ \\
$H_{20}$ & $360 \mathrm{~m}$ \\
$L$ & $663 \mathrm{~km}^{-3}$ \\
$\bar{\rho}_{10}$ & $1023.5 \mathrm{~kg} \mathrm{~m}^{-3}$ \\
$\bar{\rho}_{2}$ & $1025.0 \mathrm{~kg} \mathrm{~m}^{-3}$ \\
$\bar{\rho}_{3}$ & $1027.0 \mathrm{~kg} \mathrm{~m}^{-3}$ \\
$\Delta \rho$ & $1.4 \mathrm{~kg} \mathrm{~m}^{-3}$ \\
$y_{0}$ & $23^{\circ} \mathrm{N}$ \\
\hline
\end{tabular}

where $\bar{\rho}_{10}$ is the meridional-mean density in layer one, and $\Delta \rho$ gives the density jump across the STCC. Notice that once $U_{i}(y)$ and $\bar{\rho}_{1}(y)$ are specified, the mean layer thickness profiles $H_{i}(y)$ can be found by integrating the following coupled geostrophic balance equations:

$$
\begin{aligned}
f U_{1} & =\frac{g}{\rho_{0}} \frac{\partial}{\partial y}\left(H_{1} \bar{\rho}_{13}+H_{2} \bar{\rho}_{23}-\frac{H_{1}}{2} \frac{\partial \bar{\rho}_{1}}{\partial y}\right) \quad \text { and } \\
f U_{2} & =\frac{g}{\rho_{0}} \bar{\rho}_{23} \frac{\partial}{\partial y}\left(H_{1}+H_{2}\right)
\end{aligned}
$$

which are subject to two integration constants $H_{i 0}$, representing the $i$ th layer meridional-mean thickness. In Table 1 , we list the parameter values appropriate for the 2.5layer STCC-NEC system under the March condition.

By assuming all perturbation variables $u_{i}, v_{i}, h_{i}$, and $\rho_{1}$ have normal-mode solutions proportional to exp $i k(x-c t)$, where $k$ is the zonal wavenumber and $c\left(=c_{r}+i c_{i}\right)$ is the complex phase velocity, we can rewrite Eqs. (5)-(8) into a coupled set of differential equations in $y$ alone. Converting these differential equations into their difference forms allows us to numerically solve $c(k)$ as an eigenvalue problem. Figure 9a shows the growth rate $k c_{i}$ as a function of the zonal wavenumber $k$, with the $U_{i}(y)$ and $\bar{\rho}_{1}(y)$ profiles given by Eqs. (9) and (10) and the parameter values listed in Table 1 . In the figure, the density jump $\Delta \rho$ is changed parametrically from 0 to $1.6 \mathrm{~kg} \mathrm{~m}^{-3}$. When $\Delta \rho=0$, the most unstable wave has a wavelength of $200 \mathrm{~km}$ and an $e$-folding time scale of 40 days. These values agree qualitatively with those of the most unstable waves found in our previous 2.5-layer model study that allowed for no meridional density change in the upper layer ${ }^{1}$ and corresponded to the characteristics of the interior baroclinic instability (Qiu 1999).

\footnotetext{
${ }^{1}$ The quantitative difference is because $U_{i}(y)$ in Qiu (1999) is assumed to have constant values so that the dispersion relation $c(k)$ can be solved analytically.
}

When the meridional density gradient exists in the upper layer, Fig. 9a shows that a new type of instability with the most unstable wavelength at $30 \sim 50 \mathrm{~km}$ starts to emerge. This short-wave instability is known as the frontal, or mixed layer, instability in the existing literature (e.g., McCreary et al. 1991; Fukamachi et al. 1995; Spall 1995; Boccaletti et al. 2007; Fox-Kemper et al. 2008; Mensa et al. 2013). In Fig. 9a, the growth rate of the frontal instability becomes larger than the longer wavelength interior instability when $\Delta \rho$ exceeds $0.4 \mathrm{~kg} \mathrm{~m}^{-3}$. At the March $\Delta \rho$ value of $1.4 \mathrm{~kg} \mathrm{~m}^{-3}$ across the STCC, the most unstable frontal wave has a zonal wavelength of $\sim 50 \mathrm{~km}$ and an $e$-folding time scale of $\sim 8$ days. This wavelength is much smaller than the one related to the interior baroclinic instability. The 8-day $e$-folding time, on the other hand, is 5 times smaller than the one related to the interior instability and highlights the dominance of wintertime frontal instability in transforming potential energy into kinetic energy in the submesoscale ranges. Figure 9a reveals that the growth rate of the most unstable wave increases with the magnitude of the horizontal density gradient, ${ }^{2}$ whereas the corresponding zonal wavelength only increases slightly. This result is consistent with the previous analyses by Nakamura (1988) and Fukamachi et al. (1995).

Figures $9 \mathrm{~b}$ and $9 \mathrm{c}$ show the growth rate of the frontal instability as a function of the speed of the STCC $\left(A_{1}\right)$ and the mean upper-layer density $\left(\bar{\rho}_{10}\right)$ when $\Delta \rho$ is fixed at $1.4 \mathrm{~kg} \mathrm{~m}^{-3}$. An increase in either $A_{1}$ or $\bar{\rho}_{10}$ (which leads to a reduction in stratification between the upper two layers) enhances the growth rate, although the wavelength of the most unstable frontal instability is less affected. In Fig. 9d, we plot the frontal instability growth rate when the mean upper-layer thickness $\left(H_{1}\right)$ is changed parametrically. An increase in $H_{1}$ elevates the available potential energy (APE) stored in the upper layer and is therefore favorable for a faster-growing frontal instability. The "red" shift of the most unstable wavelength as $H_{1}$ increases in Fig. 9d is due to the increase of the Rossby radius of deformation.

To clarify further the differences between the interior and frontal unstable modes, we plot in Figs. 10a and 10b the upper- and lower-layer perturbation velocity vector and pressure distributions for the most unstable wave when $\Delta \rho=0$. In this interior instability case, the velocity and pressure perturbations have an $\mathrm{x}-\mathrm{y}$ aspect ratio of 0.5 . Vertically, the pressure perturbations are tilted

\footnotetext{
${ }^{2}$ Like in other stability analysis, the density gradient shown in Fig. 4 could be underestimated due to the occurrence of instability itself. To avoid this uncertainty, we alter $\Delta \rho$ in Fig. 9a over a broad parameter range.
} 
(a) $\Delta \rho$ Dependence

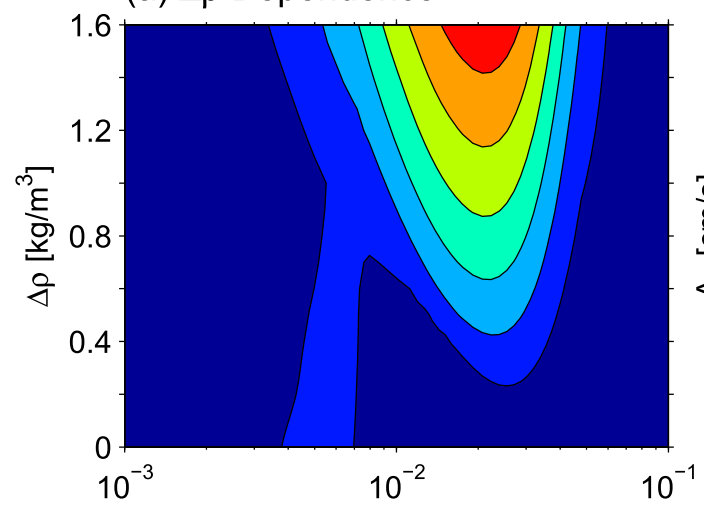

(c) Mean $\rho_{1}$ Dependence

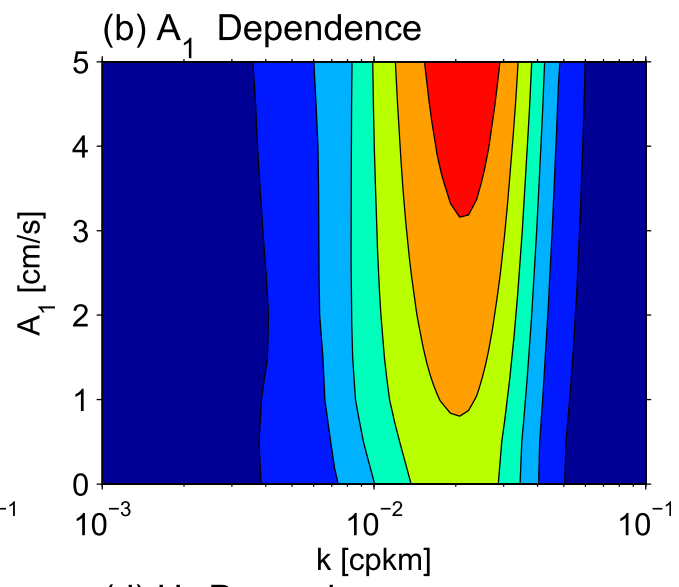

(d) $\mathrm{H}_{1}$ Dependence

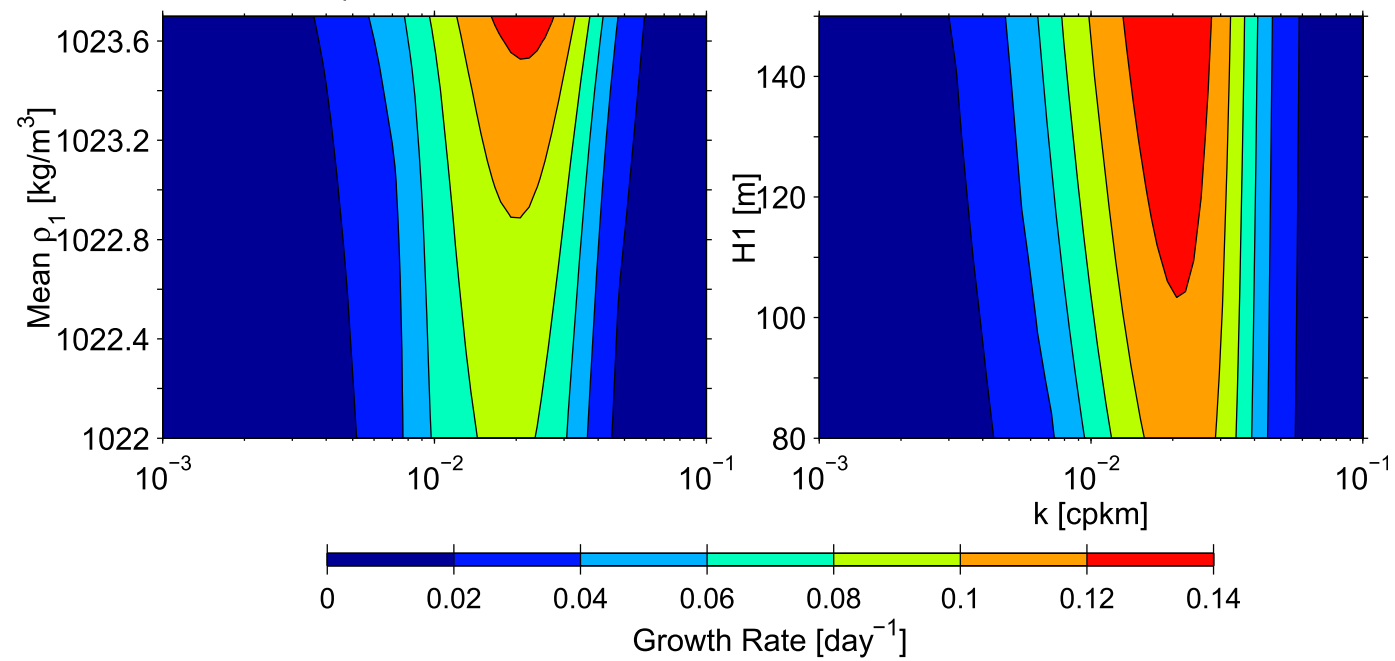

FIG. 9. (a) Growth rate $k c_{i}$ in the 2.5-layer STCC-NEC reduced-gravity model as a function of zonal wavenumber $k$ and density jump $\Delta \rho$. (b) As in (a), but for the upper-layer maximum STCC speed $A_{1}$. (c) As in (a), but for the upperlayer mean density $\bar{\rho}_{10}$. (d) As in (a), but for the upper-layer mean thickness $H_{10}$. In this last case, $H_{10}+H_{20}=460 \mathrm{~m}$ is kept as a constant.

upward toward the west, against the eastward shear of the background STCC-NEC system. Such a vertically tilted perturbation pressure signal is indicative of baroclinic instability and has been frequently detected in in situ observations in the STCC region (e.g., Roemmich and Gilson 2001; Qiu and Chen 2010). Indeed, an energetics analysis for this $\Delta \rho=0$ case confirms that the energy source for the most unstable wave comes nearly exclusively from the conversion of the background APE, that is, the $C_{3}$ term in Fig. 10c. Contributions from energy conversions due to barotropic and Kelvin-Helmholtz instabilities are minimal (see the appendix for the energetics analysis in the 2.5layer, reduced-gravity model and definitions for the energy conversion terms $C_{n}$ between the mean and eddy fields).

For the most unstable wave in the $\Delta \rho=1.4 \mathrm{~kg} \mathrm{~m}^{-3}$ case, Figs. 11a and $11 \mathrm{~b}$ reveal that the perturbation velocities are mostly confined to the upper layer (note that the vector scale in Fig. $11 \mathrm{~b}$ is 20 times smaller than in Fig. 11a). Compared to the $\Delta \rho=0$ case, the perturbations in Fig. 11 have an $x-y$ aspect ratio of 0.2 and are meridionally more elongated. As indicated by the superimposed velocity and density anomalies in Fig. 11a, the upper-layer perturbation velocity works to carry lighter and denser upper-layer water across the density gradient. This releases the background APE stored in the $y$-dependent upper-layer density field, providing the energy source for the growth of upper layer-confined frontal instability. The energetics analysis confirms this visual inspection; as shown in Fig. 11c, the energy conversion term $C_{4}$, which quantifies the energy conversion from APE of the upper-layer density gradient to eddy perturbations, is positive and takes over the $C_{3}$ term that draws the APE from the sloping isopycnals of the upper and lower layers (or the vertical shear of the zonal-mean STCC and NEC). 


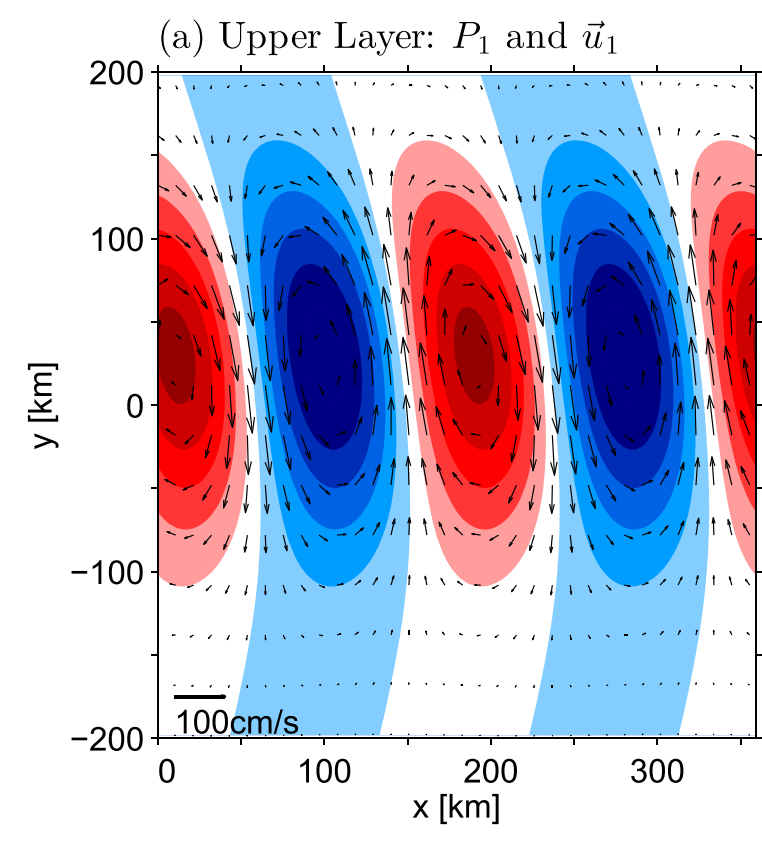

(a) Lower Layer: $P_{2}$ and $\vec{u}_{2}$

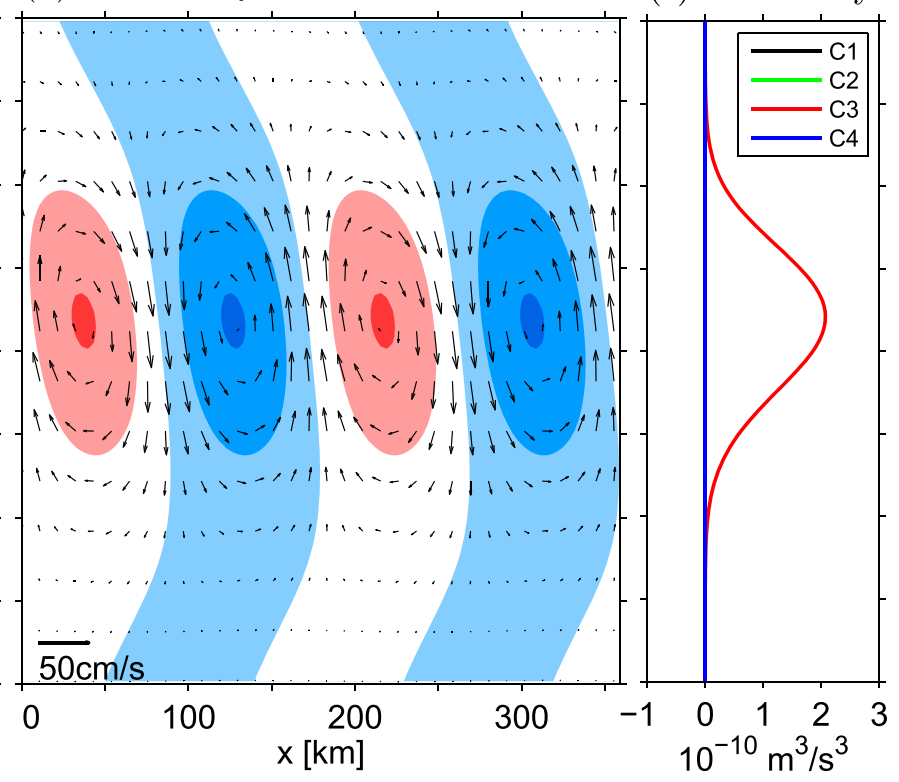

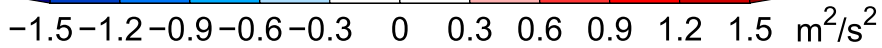

FIG. 10. (a) Eigenfunction patterns of $P_{1}$ (contours), $u_{1}$, and $v_{1}$ (vectors) for the most unstable mode when $\Delta \rho=0$. (b) As in (a), but for $P_{2}$, $u_{2}$, and $v_{2}$. (c) Conversion rates as a function of $y$ for the most unstable mode when $\Delta \rho=0$.

In concluding this section, we note that in their comparative analyses of the frontal instability, Fukamachi et al. (1995) found that using a layered model instead of a continuously stratified model as adopted in Stone (1966) and Boccaletti et al. (2007) can lead to some differences in the growth rates for small-scale perturbations. However, the similarities between the two models, in particular in terms of the growth rate magnitude, indicate that they represent basically the same physical processes of the ageostrophic frontal instabilities. The results of Fukamachi et al. (1995) lend support to the density-dependent, 2.5-layer model used in the present study, since our goal is to better contrast the relative impacts of the frontal versus interior baroclinic instabilities.

\section{Discussion}

In the primitive equation OFES model, energy conversion from the APE of the background mean state to the growth of eddy perturbations is given by $-\rho^{\prime} w^{\prime}$, where the prime denotes the deviation from the temporal mean. Figure 12a shows the $-\left\langle\rho^{\prime} w^{\prime}\right\rangle$ time series at depths of 50 versus $120 \mathrm{~m}$, where \langle\rangle denote the average in the STCC band of our interest. In Fig. 12b, we plot $-\left\langle\rho^{\prime} w^{\prime}\right\rangle$ as a function of depth in which the black line denotes the mixed layer depth averaged in the same STCC band. There are two noteworthy features in
Fig. 12. First, during the developing phase of the winter mixed layer from December to March, the baroclinic energy conversion $-\left\langle\rho^{\prime} w^{\prime}\right\rangle$ is largely confined to the surface mixed layer and its amplitude increases with the deepening of the mixed layer. Once the mixed layer starts to shoal after mid-March, the APE source for the frontal instability weakens and so does the baroclinic energy conversion in the surface mixed layer. In contrast to the mixed layer $-\left\langle\rho^{\prime} w^{\prime}\right\rangle$ signals, the baroclinic energy conversion starts to gain strength in the interior ocean after March. At the 120-m depth, for example, Fig. 12a reveals that $-\left\langle\rho^{\prime} w^{\prime}\right\rangle$ has a maximum in mid-April, lagging behind the mixed layer energy conversion peak by 1 month.

The OFES-simulated $-\left\langle\rho^{\prime} w^{\prime}\right\rangle$ of Fig. 12 and the EKE characteristics shown in Fig. 5 can be interpreted within the framework of two types of instabilities explored in section 4 using the simplified 2.5-layer, reduced-gravity model. Specifically, during the developing phase of the winter mixed layer, the frontal instability dominates and its intensity increases with the winter months because of the progressive increase in $\Delta \rho$ in the mixed layer across the STCC (recall Fig. 4). As the frontal instability in the STCC system has a weekly $e$-folding time scale, the submesoscale EKE evolution (i.e., the solid red line in Fig. 5b) exhibits a time series very similar to that of $-\left\langle\rho^{\prime} w^{\prime}\right\rangle$ within the mixed layer (cf. the red line in Fig. 12a).

Concurrent with the frontal instability of the surface mixed layer, interior baroclinic instability occurs at the 
(a) Upper Layer: $\rho_{1}$ and $\vec{u}_{1}$

(b) Lower Layer: $P_{2}$ and $\vec{u}_{2}$

(c) Instability

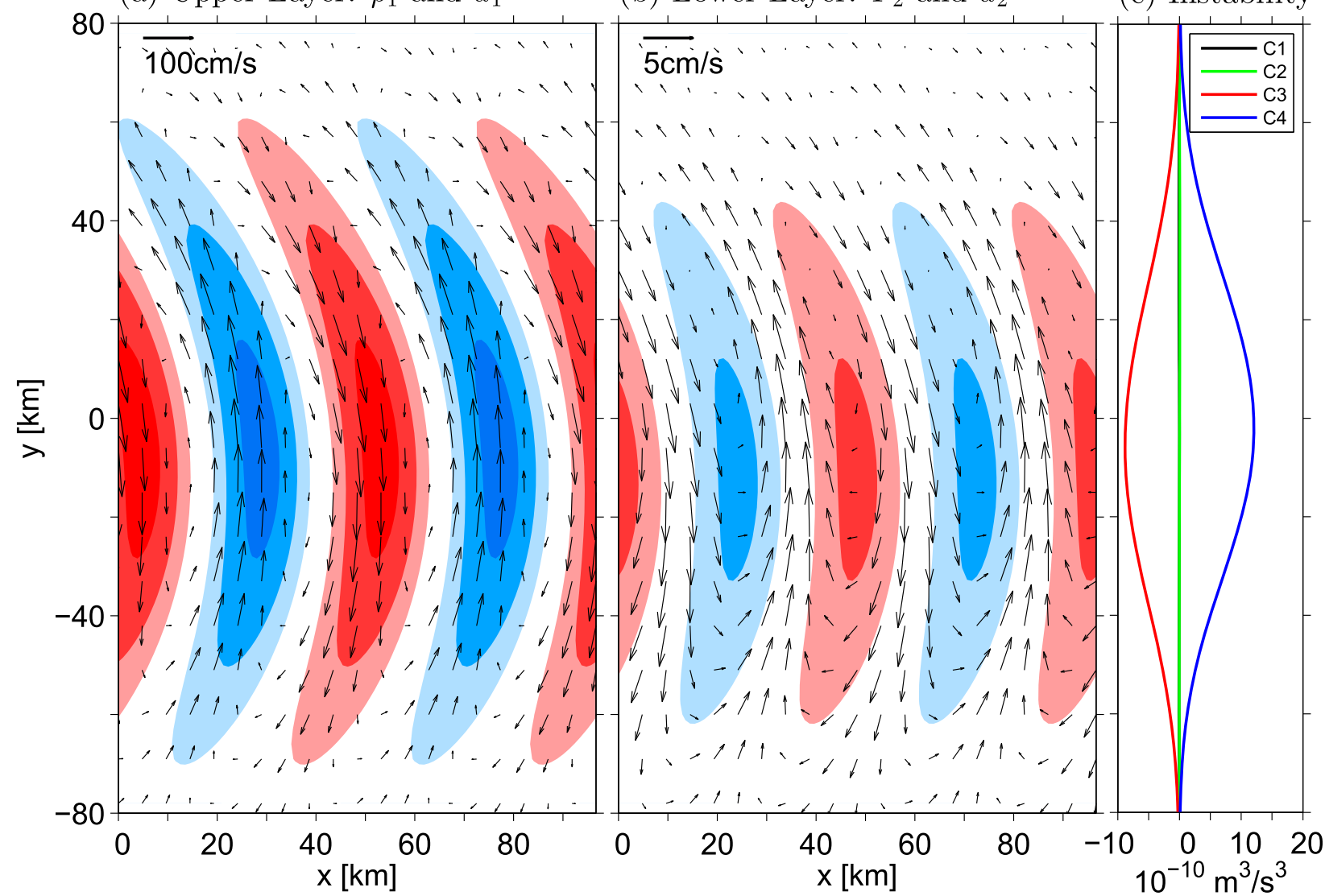

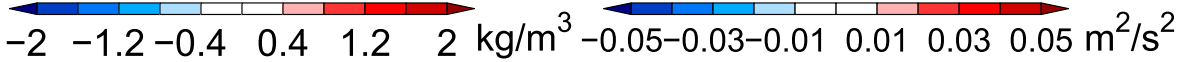

FIG. 11. As in Fig. 10, but for $\Delta \rho=1.4 \mathrm{~kg} \mathrm{~m}^{-3}$.

expense of the vertical shear of the STCC-NEC system. With the $e$-folding time scale at $\sim 40$ days, this slowgrowing interior baroclinic instability is responsible for $-\left\langle\rho^{\prime} w^{\prime}\right\rangle$ that penetrate deep below the winter mixed layer and have an energy conversion peak that lags behind the mixed layer frontal instability. The most unstable perturbations of the interior baroclinic instability have a wavelength of $O(200) \mathrm{km}$, and the deep-reaching mesoscale EKE signals shown in Fig. 5d are largely a consequence of this interior baroclinic instability.

Since most OGCM and climate models do not resolve the ageostrophic frontal instability directly, a parameterization scheme has been proposed recently by FoxKemper et al. $(2008,2011)$ to incorporate the $-\left\langle\rho^{\prime} w^{\prime}\right\rangle$ effects of the surface mixed layer. By introducing an overturning streamfunction, they demonstrate that the baroclinic energy conversion averaged in the mixed layer is proportional to

$$
-\left\langle\rho^{\prime} w^{\prime}\right\rangle_{\mathrm{ML}} \propto\left\langle\left|\nabla \bar{\rho}_{\mathrm{ML}}\right|\right\rangle^{2} \times\left\langle D_{\mathrm{ML}}\right\rangle^{2}
$$

where $\nabla \bar{\rho}_{\mathrm{ML}}$ is the horizontal gradient of mixed layer density, and $D_{\mathrm{ML}}$ is the mixed layer depth. To test this parameterization scheme, we plot in Fig. 13a the time series of $\left\langle\left|\nabla \bar{\rho}_{\mathrm{ML}}\right|\right\rangle^{2}$ and $\left\langle D_{\mathrm{ML}}\right\rangle^{2}$ averaged in the STCC band. The product of these time series is shown by the red line in Fig. 13b. Compared to the $-\left\langle\rho^{\prime} w^{\prime}\right\rangle_{\mathrm{ML}}$ time series denoted by blue line, the parameterization scheme works quite favorably in the late fall to spring seasons. Between the two contributing terms, it is the squared, mixed layer depth change that controls the RHS of Eq. (11), a result consistent with the findings of Capet et al. (2008a) and Mensa et al. (2013). For the STCC region of our interest, the parameterization scheme works less favorably during July-October. Instead of the frontal instability, the baroclinic energy conversion in the mixed layer during these months is controlled by the synoptic surface wind forcing associated with typhoons and tropical cyclones in the northwestern Pacific Ocean (e.g., Qiu et al. 2004). 
(a) $-<\rho ' w^{\prime}>$ at $50 m$ and $120 m$

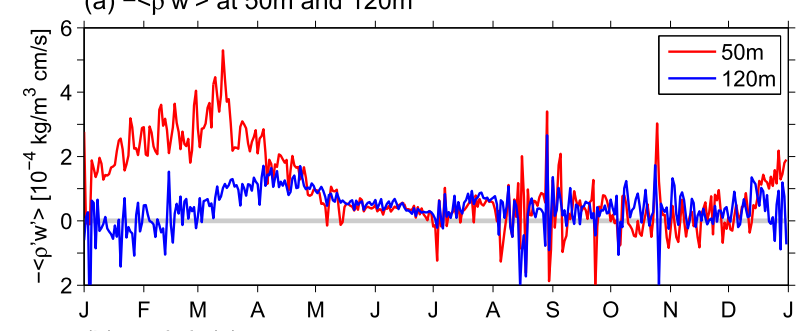

(b) $-<\rho ' w^{\prime}>(z)$

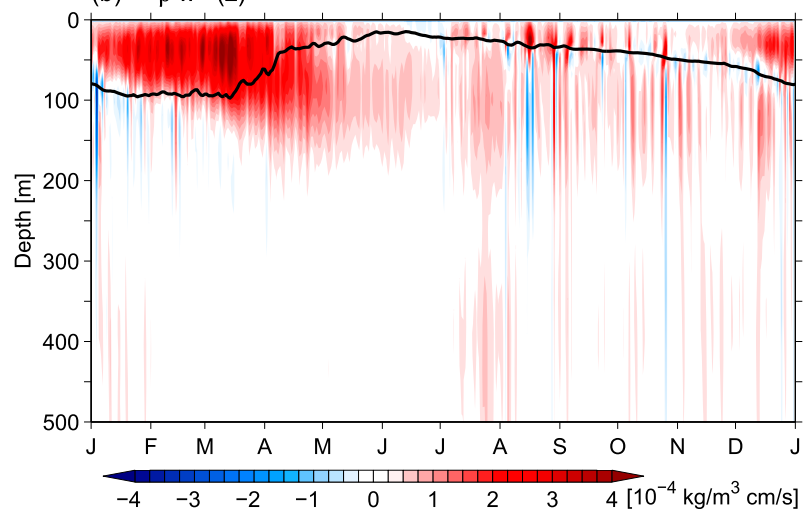

FIG. 12. (a) Time series of the baroclinic conversion rate $-\left\langle\rho^{\prime} w^{\prime}\right\rangle$ at the 50- and $120-\mathrm{m}$ depths in the STCC band of $18^{\circ}-28^{\circ} \mathrm{N}$ and $135^{\circ}-160^{\circ}$ E. (b) The $-\left\langle\rho^{\prime} w^{\prime}\right\rangle$ as a function of depth. Black line denotes the mixed layer depth in the same STCC band.

It is important to emphasize that linear instability analyses presented in section 4 provide only the initial eddy length scales. As the perturbations grow in amplitude, nonlinear interactions can modify the dominating eddy scales through energy cascade (e.g., Rhines 1977). Indeed, rather than a step function-like time evolution involving the two most unstable wavelengths of frontal and interior baroclinic instabilities, Fig. 7a reveals that the energy-containing length scale in the OFES simulation modulates gradually as the season progresses. To quantify the energy cascade processes simulated in OFES, we evaluate the surface spectral KE flux defined by

$$
\Pi(K)=-\int_{K}^{K_{N}} \Re\left(\hat{u}^{*} u_{j} \frac{\partial u}{\partial x_{j}}+\hat{v}^{*} u_{j} \frac{\partial v}{\partial x_{j}}\right) d K
$$

where $(u, v) \equiv\left(u_{1}, u_{2}\right)$ are the surface zonal and meridional velocities, carets indicate discrete Fourier transform, asterisks indicate complex conjugates, $K=\sqrt{k^{2}+l^{2}}$ is the total wavenumber, and $K_{N}$ is the Nyquist total wavenumber of the OFES model grid. In Eq. (12), summations over the repeat index $j$ are assumed. Physically, a positive $\Pi\left(K_{0}\right)$ signifies a forward KE flux from wavenumber $K<$ $K_{0}$ to $K>K_{0}$, and a negative $\Pi\left(K_{0}\right)$ is indicative of an inverse $\mathrm{KE}$ cascade from $K>K_{0}$ to $K<K_{0}$. Because the rate of spectral $\mathrm{KE}$ change is relatively small, a positive spectral KE flux divergence, $\partial \Pi / \partial K>0$, at $K_{0}$ often implies the presence of an energy source at that wavenumber (Scott and Wang 2005; Qiu et al. 2008).

Figure 14 a shows the monthly $\Pi(K)$ distribution when the OFES SSH output is used in calculating Eq. (12). For comparison, we plot in Fig. 14b the same flux distribution inferred from the monthly AVISO SSH data of the past 20 yr. From Fig. 14a, it is clear that the spectral KE flux is mostly negative, indicating the prevalence of the inverse $\mathrm{KE}$ cascade for both the mesoscale and submesoscale wavenumbers. The amplitude of $\Pi(K)$ is, however, seasonally dependent; enhanced inverse KE cascade emerges from January to June, and a seasonal peak occurs in March when the frontal instability is at maximum. During March, Fig. 14a reveals that $\partial \Pi / \partial K$ is positive for $5.5 \times 10^{-3}<K<4.0 \times 10^{-2} \mathrm{cpkm}$. With positive $\partial \Pi / \partial K$ implying the presence of an external energy source, it is not coincident that this is the wavenumber window within which the frontal instability is most vigorous (recall Fig. 9). Notice that the short-wave cutoff for the inverse KE cascade in January-March has a wavelength of $38 \mathrm{~km}$, which is much shorter than $2 \pi$ times the local deformation radius, $\sim 310 \mathrm{~km}$, in the STCC region. That the inverse KE cascade can extend well into the submesoscale wavenumber range when the frontal instability is operating has also been found in other recent high-resolution modeling studies (e.g., Capet et al. 2008c; Klein et al. 2008). Following the weakening of frontal instability in April, Fig. 14a shows that the short-wave cutoff for the inverse KE cascade tends to shift gradually to a smaller wavenumber.

Several studies in the past have utilized satellite altimetry data to infer the spectral KE fluxes (e.g., Scott and Wang 2005; Qiu et al. 2008; Tulloch et al. 2011; Arbic et al. 2013). While the AVISO-derived $\Pi(K)$ captures the inverse $\mathrm{KE}$ cascade at the mesoscale wavenumber range of $K<4 \times 10^{-3}$ cpkm (see Fig. 14b) for the STCC region, the lack of submesoscale SSH information in the AVISO product causes its amplitude to be underestimated (notice the difference in contour intervals between Figs. 14a and 14b) and its seasonal peak to emerge delayed when compared with the OFES result (i.e., May as compared to March). It is important to emphasize that the intense forward KE cascade seen in Fig. $14 \mathrm{~b}$ for $K>4 \times 10^{-3} \mathrm{cpkm}$ is an artifact of the objectively mapped AVISO product. In fact, when $\eta_{\mathrm{MS}}^{\prime}$, instead of $\eta^{\prime}$, in Eq. (2) from the OFES simulation was used to evaluate the spectral KE fluxes (see Fig. 14c), a positive $\Pi(K)$ signal similar to that in Fig. 14b emerges in the high-wavenumber range. Also distorted in Fig. 14c is the seasonal variation in the short-wave cutoff for the inverse KE cascade that exists in the original OFES result 

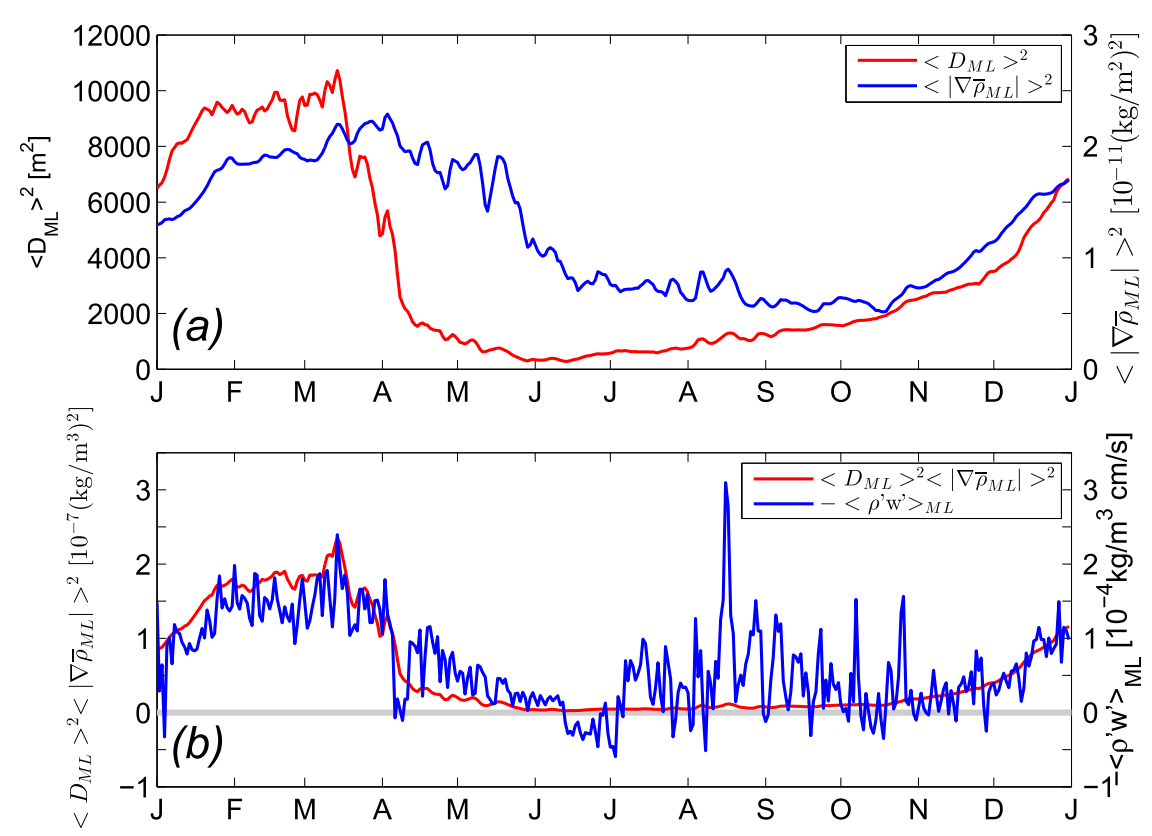

FIG. 13. (a) Time series of squared horizontal mixed layer density gradient $\left\langle\left|\nabla \bar{\rho}_{\text {ML }}\right|\right\rangle^{2}$ (blue) and mixed layer depth $\left\langle D_{\mathrm{ML}}\right\rangle^{2}$ (red) averaged in the STCC band of $18^{\circ}-28^{\circ} \mathrm{N}$ and $135^{\circ}-160^{\circ} \mathrm{E}$. (b) Time series of $\left\langle\left|\nabla \bar{\rho}_{\mathrm{ML}}\right|\right\rangle^{2} \times\left\langle D_{\mathrm{ML}}\right\rangle^{2}$ (red) vs the baroclinic conversion rate $-\left\langle\rho^{\prime} w^{\prime}\right\rangle_{\mathrm{ML}}$ averaged in the mixed layer (blue).

of Fig. 14a; as in the AVISO result, the short-wave cutoff in Fig. 14c exhibits little seasonal dependence. The issue of spurious $\Pi(K)>0$ signals in the high-wavenumber range was also raised recently by Arbic et al. (2013), and caution is clearly called for in interpreting the forward $\mathrm{KE}$ cascade inferred from the gridded AVISO product.

\section{Summary}

With the use of a $1 / 30^{\circ}$-resolution North Pacific OGCM simulation (OFES), we have in this study examined the seasonal mesoscale and submesoscale eddy variability that coexists along the Subtropical Countercurrent band of $18^{\circ}-28^{\circ} \mathrm{N}$ in the western North Pacific Subtropical Gyre. Much of our current knowledge about the STCC's seasonal eddy variability has been based on the nadirlooking satellite altimeter measurements from which the widely used AVISO SSH product is derived. The spatial SSH signals resolvable by AVISO are nominally larger than $O(150) \mathrm{km}$. Because of the lack of SSH information with length scales $<150 \mathrm{~km}$, we found in this study that our understanding of the seasonal STCC eddy variability is both incomplete and, in some aspects, distorted.

Phenomenologically, while the AVISO product detects the seasonal EKE and rms vorticity maxima in May and April, respectively, in the STCC band, the OFES simulation indicates that both of these maxima appear in March. In terms of the surface vorticity asymmetry, the
AVISO product reveals a weak positive skewness of $\sim 0.2$ without a clear seasonal dependence. In comparison, the OFES simulation points to a much larger positive skewness of $\sim 0.7$ and a pronounced seasonality that peaks in March and April. With regards to the eddyeddy interactions, the AVISO-inferred spectral kinetic energy flux shows an inverse $\mathrm{KE}$ cascade for wavelengths larger than $250 \mathrm{~km}$ and a forward KE cascade for wavelengths shorter than $250 \mathrm{~km}$. The inferred spectral fluxes have a seasonal maximum in May. In contrast, the OFES simulation reveals that the short-wave cutoff for the inverse KE cascade migrates seasonally between 35 and $100 \mathrm{~km}$. The most intense inverse KE cascade takes place in March when the short-wave cutoff extends furthest into the $35-\mathrm{km}$ wavelength.

These phenomenological differences between the AVISO and OFES results stem from the fact that two types of baroclinic instabilities are concurrently occurring in the seasonally modulating STCC-NEC system. With the aid of a 2.5-layer, reduced-gravity model with an embedded surface-layer density gradient that mimics the STCC-NEC system, we showed that the first baroclinic instability is of interior type and has its energy source in available potential energy of the oppositeflowing STCC-NEC system. The most unstable waves of the interior baroclinic instability have, in March, a growth time scale of $O(40)$ days and a wavelength of $O(250) \mathrm{km}$, and these unstable wave characteristics change with 
(a) OFES STCC $\Pi$

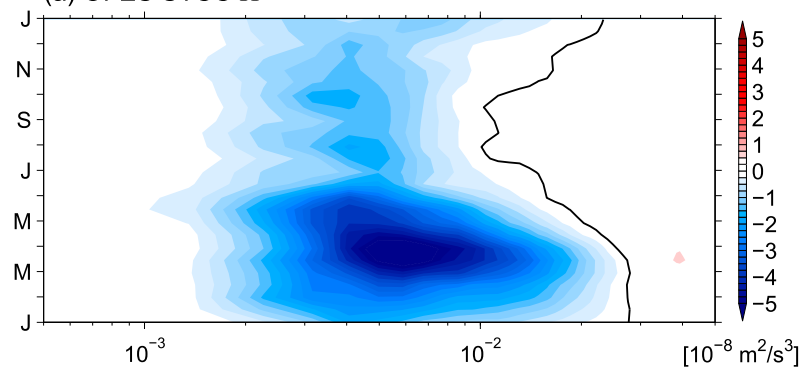

(b) AVISO (climatology) STCC ח

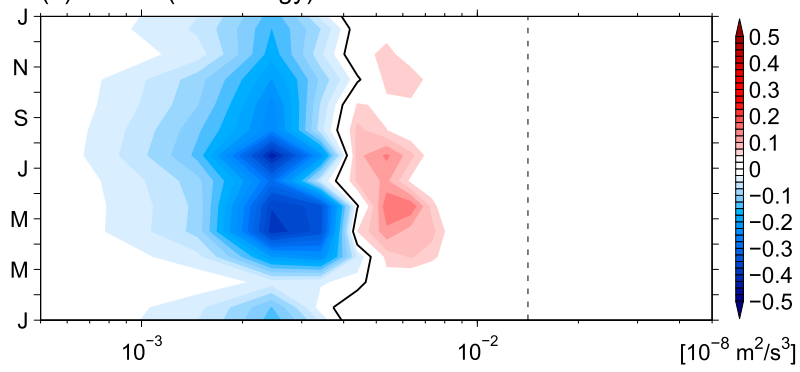

(c) OFES STCC П (MS)

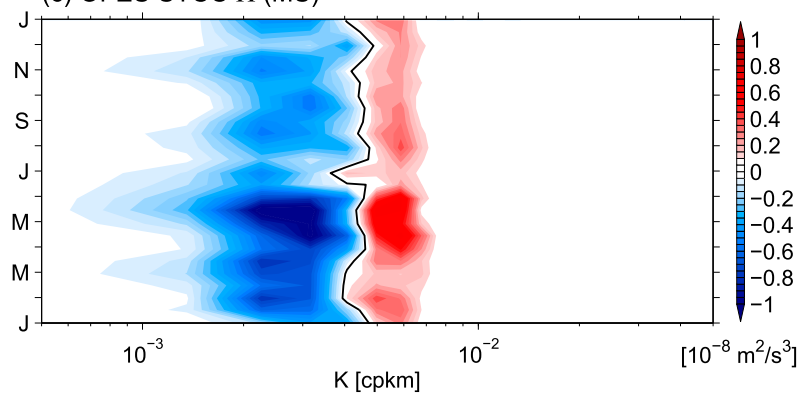

FIG. 14. (a) Monthly spectral kinetic energy flux $\Pi(K)$ distribution based on the OFES-simulated SSH data $\eta^{\prime}$. Black contour denotes $\Pi(K)=0$. (b) As in (a), but for the AVISO gridded SSH data. Dashed line denotes the Nyquist wavenumber of the AVISO data. (c) As in (a), but based on the OFES-simulated mesoscale SSH data $\eta_{\text {MS }}^{\prime}$ [see Eq. (2)]. Note that contour intervals are different among the three plots.

the seasonally evolving STCC-NEC background flows. The interior baroclinic instability has been identified in the previous studies to be responsible for the seasonal EKE modulations detected based on the AVISO SSH data.

The second type of baroclinic instability derives its energy from the meridional density gradient across the STCC in the deep winter mixed layer, and the instabilityinduced perturbations are surface confined. The most unstable wave of the frontal baroclinic instability has a short wavelength of $\sim 50 \mathrm{~km}$ and a rapid growth on a weekly time scale. While potent in its growth, on the other hand, the frontal baroclinic instability is temporally restricted; its APE source depletes quickly once the surface mixed layer starts to shoal after mid-March. In comparison, despite its seasonal modulation, the vertical shear of the STCC-NEC system that provides the energy source for the interior baroclinic instability persists throughout the year. It is important to note that due to the inverse KE cascade, the mesoscale EKE signals are seasonally controlled by both the interior baroclinic instability and the upscale energy fluxes resulting from the frontal baroclinic instability. It is by these characteristics of the two competing baroclinic instabilities that the seasonal mesoscale and submesoscale eddy modulations in the STCC region are ultimately determined.

It is natural to ask if improving the OFES model resolution would further alter the seasonal EKE signals along the STCC band. With respect to the timing of its seasonality, we believe that increasing the model resolution beyond $3 \mathrm{~km}$ will not significantly shift the seasonal EKE peak to an earlier date. The reasons for this are twofold. First, the timing for the maximum mixed layer depth and upper-ocean density gradient in March is set by the surface atmospheric forcing and not by internal ocean dynamics. Since the growth time for the frontal/mixed layer instability is only a few days, allowing for faster-growing, smaller-scale disturbances will not change the March EKE peak. Second, existing high-resolution OGCM simulations reveal that the upper-ocean EKE spectrum has a $k^{-2}$ slope. With their diminished kinetic energy level, the smaller-scale eddies of $O(1) \mathrm{km}$ are unlikely to modify the seasonality of the EKE time series in a significant way.

In closing, we note that we have in this study relied on the high-resolution OFES simulation to explore the seasonal submesoscale eddy features and their impact on the mesoscale eddy field. Based on the wide-swath satellite interferometry, National Aeronautics and Space Administration (NASA) and Centre National d'Etudes Spatiales (CNES) are at present jointly developing the Surface Water and Ocean Topography (SWOT) mission to measure the global SSH field with a spatial resolution of $O(10) \mathrm{km}$ (Fu and Ferrari 2008; http://swot.jpl.nasa.gov/). A similar interferometry mission, named the Coastal and Ocean Measurement Mission with Precise and Innovative Radar Altimeter (COMPIRA), is also pursued currently by Japan Aerospace Exploration Agency (JAXA; Uematsu et al. 2013). With their launches scheduled in 2020, the SWOT and COMPIRA missions will be highly relevant to verify the new mesoscale and submesoscale features of the STCC identified in this study and to improve our understanding of the mesoscale-submesoscale interaction processes that a high-resolution numerical model, such as OFES, may have inadequately simulated.

Acknowledgments. We thank Lee Fu, Jay McCreary, and Shafer Smith for fruitful discussions. Detailed and constructive comments made by Baylor Fox-Kemper and an anonymous reviewer helped improve an early version of the manuscript. The satellite altimeter products 
are provided by Ssalto/Duacs and distributed by AVISO with support from CNES. B.Q. and S.C. acknowledge support from NASA SWOT and OSTST missions (NNX13AD91G and NNX13AE51E). P.K. acknowledges support of CNRS (France) and Agence Nationale pour la Recherche [ANR-09-BLAN-0365-02 (REDHOT) and ANR-10-LABX-19-01 (LabexMER) ]. H.S. and Y.S. are supported by MEXT/JST KAKENHI (25400473 and 22106006).

\section{APPENDIX}

\section{Energetics Analysis in a 2.5-Layer Model with $\bar{\rho}_{1}(y)$}

Following McCreary et al. (1991), the sum of the eddy kinetic and potential energy in the 2.5-layer, reducedgravity model shown in Fig. 8 can be written as

$$
\begin{aligned}
\tilde{E}= & \frac{1}{2} H_{i}\left\langle u_{i}^{2}+v_{i}^{2}\right\rangle+U_{i}\left\langle h_{i} u_{i}\right\rangle \\
& -\frac{g}{2 \rho_{0}}\left[\bar{\rho}_{12}\left\langle h_{1}^{2}\right\rangle+\bar{\rho}_{23}\left\langle\left(h_{1}+h_{2}\right)^{2}\right\rangle+2 H_{1}\left\langle h_{1} \rho_{1}\right\rangle\right],
\end{aligned}
$$

where \langle\rangle denote averaging over a zonal wavelength $2 \pi / k$ and summations over the repeated layer index $i$ are assumed. The temporal change of $\tilde{E}$ due to the interaction of eddy and mean fields is given by $\partial \tilde{E} / \partial t=\sum_{n=1}^{4} C_{n}$, where the four conversion terms are

$$
\begin{aligned}
C_{1}= & -\left(H_{i}\left\langle u_{i} v_{i}\right\rangle+U_{i}\left\langle h_{i} v_{i}\right\rangle\right) \frac{\partial U_{i}}{\partial y}, \\
C_{2}= & -U_{i}\left\langle u_{i}\left[\frac{\partial\left(H_{i} u_{i}\right)}{\partial x}+\frac{\partial\left(H_{i} v_{i}\right)}{\partial y}\right]\right\rangle, \\
C_{3}= & \frac{g}{\rho_{0}}\left\langle h_{1} v_{1}\right\rangle\left(\bar{\rho}_{13} \frac{\partial H_{1}}{\partial y}+\bar{\rho}_{23} \frac{\partial H_{2}}{\partial y}\right) \\
& +\frac{g}{\rho_{0}}\left\langle h_{2} v_{2}\right\rangle \bar{\rho}_{23} \frac{\partial}{\partial y}\left(H_{1}+H_{2}\right), \text { and } \\
C_{4}= & \frac{g}{\rho_{0}}\left\langle h_{1} v_{1}\right\rangle H_{1} \frac{\partial \bar{\rho}_{1}}{\partial y}+\frac{g}{2 \rho_{0}} H_{1} U_{1}\left\langle h_{1} \frac{\partial \rho_{1}}{\partial x}\right\rangle \\
& -\frac{g}{\rho_{0}} H_{1} U_{1}\left\langle\frac{\partial}{\partial x}\left(h_{1} \rho_{1}\right)\right\rangle \\
& +\frac{g}{2 \rho_{0}}\left\langle\rho_{1}\left[\frac{\partial}{\partial x}\left(H_{1}^{2} u_{1}\right)+\frac{\partial}{\partial y}\left(H_{1}^{2} v_{1}\right)\right]\right\rangle .
\end{aligned}
$$

Physically, a positive $C_{1}$ signifies barotropic instability, a positive $C_{2}$ signifies a Kelvin-Helmholtz instability, a positive $C_{3}$ signifies a baroclinic instability, and a positive $C_{4}$ signifies frontal instability. Notice that baroclinic instability grows by extracting mean potential energy stored in the tilting isopycnals of the layers, whereas frontal instability only occurs when the meridional density gradient is present in the upper layer.

\section{REFERENCES}

Arbic, B. K., K. L. Polzin, R. B. Scott, J. G. Richman, and J. F. Shriver, 2013: On eddy viscosity, energy cascades, and the horizontal resolution of gridded satellite altimeter products. J. Phys. Oceanogr., 43, 283-300, doi:10.1175/JPO-D-11-0240.1.

Boccaletti, G., R. Ferrari, and B. Fox-Kemper, 2007: Mixed layer instabilities and restratification. J. Phys. Oceanogr., 37, 22282250, doi:10.1175/JPO3101.1.

Capet, X., E. J. Campos, and A. M. Paiva, 2008a: Submesoscale activity over the Argentinian shelf. Geophys. Res. Lett., 35, L15605, doi:10.1029/2008GL034736.

_ J. C. McWilliams, M. J. Molemaker, and A. J. Shchepetkin, 2008b: Mesoscale to submesoscale transition in the California Current System. Part I: Flow structure, eddy flux, and observational tests. J. Phys. Oceanogr., 38, 29-43, doi:10.1175/ 2007JPO3671.1.

,,--- , and,$- 2008 \mathrm{c}$ : Mesoscale to submesoscale transition in the California Current System. Part III: Energy balance and flux. J. Phys. Oceanogr., 38, 2256-2269, doi:10.1175/ 2008JPO3810.1.

Chang, Y.-L., and L.-Y. Oey, 2014: Instability of the North Pacific Subtropical Countercurrent. J. Phys. Oceanogr., 44, 818-833, doi:10.1175/JPO-D-13-0162.1.

Chelton, D. B., R. A. de Szoeke, M. G. Schlax, K. E. Naggar, and N. Siwertz, 1998: Geographical variability of the first baroclinic Rossby radius of deformation. J. Phys. Oceanogr., 28, 433-460, doi:10.1175/1520-0485(1998)028<0433:GVOTFB > 2.0.CO;2.

_- M. G. Schlax, and R. M. Samelson, 2011: Global observations of nonlinear mesoscale eddies. Prog. Oceanogr., 91, 167-216, doi:10.1016/j.pocean.2011.01.002.

Eldevik, T., and K. B. Dysthe, 2002: Spiral eddies. J. Phys. Oceanogr., 32, 851-869, doi:10.1175/1520-0485(2002)032<0851: $\mathrm{SE}>2.0 . \mathrm{CO} ; 2$.

Fox-Kemper, B., R. Ferrari, and R. Hallberg, 2008: Parameterization of mixed layer eddies. Part I: Theory and diagnosis. J. Phys. Oceanogr., 38, 1145-1165, doi:10.1175/2007JPO3792.1.

, G. Danabasoglu, R. Ferrari, R. W. Hallberg, M. M. Holland, M. E. Maltrud, S. Peacock, and B. L. Samuels, 2011: Parameterization of mixed layer eddies. III: Implementation and impact in global ocean climate simulations. Ocean Modell., 39, 61-78, doi:10.1016/j.ocemod.2010.09.002.

Fu, L. L., and R. Ferrari, 2008: Observing oceanic submesoscale processes from space. Eos, Trans. Amer. Geophys. Union, 89, 488, doi:10.1029/2008EO480003.

— D. B. Chelton, P.-Y. Le Traon, and R. Morrow, 2010: Eddy dynamics from satellite altimetry. Oceanography, 23, 14-25, doi:10.5670/oceanog.2010.02.

Fukamachi, Y., J. P. McCreary, and J. A. Proehl, 1995: Instability of density fronts in layer and continuously stratified models. J. Geophys. Res., 100, 2559-2577, doi:10.1029/94JC02656.

Hosoda, S., T. Ohira, and T. Nakamura, 2008: A monthly mean dataset of global oceanic temperature and salinity derived from Argo float observations. JAMSTEC Rep. Res. Dev., 8, 47-59, doi:10.5918/jamstecr.8.47.

Klein, P., B. L. Hua, G. Lapeyre, X. Capet, S. Le Gentil, and H. Sasaki, 2008: Upper ocean turbulence from high-resolution 
3D simulations. J. Phys. Oceanogr., 38, 1748-1763, doi:10.1175/ 2007JPO3773.1.

Kobashi, F., and H. Kawamura, 2002: Seasonal variation and instability nature of the North Pacific Subtropical Countercurrent and the Hawaiian Lee Countercurrent. J. Geophys. Res., 107, 3185, doi:10.1029/2001JC001225.

— tropical Countercurrents and Subtropical Fronts: Role of mode waters in ocean circulation and climate. J. Oceanogr., 68, 113-126, doi:10.1007/s10872-011-0048-x.

Masumoto, Y., and Coauthors, 2004: A fifty-year eddy-resolving simulation of the World Ocean-Preliminary outcomes of OFES (OGCM for the Earth Simulator). J. Earth Simulator, 1, 35-56.

McCreary, J. P., Y. Fukamachi, and P. K. Kundu, 1991: A numerical investigation of jets and eddies near an eastern ocean boundary. J. Geophys. Res., 96, 2515-2534, doi:10.1029/ 90JC02195.

Mensa, J. A., Z. Garraffo, A. Griffa, T. M. Ozgokmen, A. Haza, and M. Veneziani, 2013: Seasonality of the submesoscale dynamics in the Gulf Stream region. Ocean Dyn., 63, 923-941, doi:10.1007/s10236-013-0633-1.

Morrow, R., and P.-Y. Le Traon, 2012: Recent advances in observing mesoscale ocean dynamics with satellite altimetry. Adv. Space Res., 50, 1062-1076, doi:10.1016/j.asr.2011.09.033.

Munk, W., L. Armi, K. Fischer, and Z. Zachariasen, 2000: Spirals on the sea. Proc. Roy. Soc. London, A456, 1217-1280, doi:10.1098/ rspa.2000.0560.

Nakamura, N., 1988: Scale selection of baroclinic instability-Effects of stratification and nongeostrophy. J. Atmos. Sci., 45, 3253-3268, doi:10.1175/1520-0469(1988)045<3253:SSOBIO > 2.0.CO;2.

Noh, Y., and H. Kim, 1999: Simulations of temperature and turbulence structure of the oceanic boundary layer with the improved near-surface process. J. Geophys. Res., 104, 1562115 634, doi:10.1029/1999JC900068.

— B. Y. Yim, S. H. You, J. H. Yoon, and B. Qiu, 2007: Seasonal variation of eddy kinetic energy of the North Pacific Subtropical Countercurrent simulated by an eddy-resolving OGCM. Geophys. Res. Lett., 34, L07601, doi:10.1029/2006GL029130.

Onogi, K., and Coauthors, 2007: The JRA-25 Reanalysis. J. Meteor. Soc. Japan, 85, 369-432, doi:10.2151/jmsj.85.369.

Qiu, B., 1999: Seasonal eddy field modulation of the North Pacific Subtropical Countercurrent: TOPEX/POSEIDON observations and theory. J. Phys. Oceanogr., 29, 2471-2486, doi:10.1175/ 1520-0485(1999)029<2471:SEFMOT>2.0.CO;2.

, and S. Chen, 2010: Interannual variability of the North Pacific Subtropical Countercurrent and its associated mesoscale eddy field. J. Phys. Oceanogr., 40, 213-225, doi:10.1175/ 2009JPO4285.1. $\longrightarrow, \ldots$, and P. Hacker, 2004: Synoptic-scale air-sea flux forcing in the western North Pacific: Observations and their impact on SST and the mixed layer. J. Phys. Oceanogr., 34, 2148-2159, doi:10.1175/1520-0485(2004)034<2148:SAFFIT>2.0.CO;2.

- R. Scott, and S. Chen, 2008: Length scales of eddy generation and nonlinear evolution of the seasonally modulated South Pacific Subtropical Countercurrent. J. Phys. Oceanogr., 38, 1515-1528, doi:10.1175/2007JPO3856.1.

Rhines, P. B., 1977: The dynamics of unsteady currents. The SeaIdeas and Observations on Progress in the Study of the Seas, E. D. Goldberg, Ed., Marine Modeling, Vol. 6, John Wiley and Sons, $189-318$.

Rio, M. H., S. Guinehut, and G. Larnicol, 2011: New CNES-CLS09 global mean dynamic topography computed from the combination of GRACE data, altimetry, and in situ measurements. J. Geophys. Res., 116, C07018, doi:10.1029/2010JC006505.

Roemmich, D., and J. Gilson, 2001: Eddy transport of heat and thermocline waters in the North Pacific: A key to interannual/ decadal climate variability? J. Phys. Oceanogr., 31, 675-687, doi:10.1175/1520-0485(2001)031<0675:ETOHAT>2.0.CO;2.

Rudnick, D., 2001: On the skewness of vorticity in the upper ocean. Geophys. Res. Lett., 28, 2045-2048, doi:10.1029/2000GL012265.

Sasaki, H., and P. Klein, 2012: SSH wavenumber spectra in the North Pacific from a high-resolution realistic simulation. J. Phys. Oceanogr., 42, 1233-1241, doi:10.1175/JPO-D-11-0180.1.

—, M. Nonaka, Y. Masumoto, Y. Sasai, H. Uehara, and H. Sakuma, 2008: An eddy-resolving hindcast simulation of the quasiglobal ocean from 1950 to 2003 on the Earth Simulator. High Resolution Numerical Modelling of the Atmosphere and Ocean, W. Ohfuchi and K. Hamilton, Eds., Springer, 157-185, doi:10.1007/ 978-0-387-49791-4_10.

Scott, R. B., and F. Wang, 2005: Direct evidence of an oceanic inverse kinetic energy cascade from satellite altimetry. J. Phys. Oceanogr., 35, 1650-1666, doi:10.1175/JPO2771.1.

Spall, M. A., 1995: Frontogenesis, subduction, and cross-front exchange at upper ocean fronts. J. Geophys. Res., 100, $2543-$ 2557, doi:10.1029/94JC02860.

Stone, P. H., 1966: On non-geostrophic baroclinic stability. J. Atmos. Sci., 23, 390-400, doi:10.1175/1520-0469(1966)023<0390: ONGBS $>2.0 . \mathrm{CO} ; 2$.

Tulloch, R., J. Marshall, C. Hill, and K. S. Smith, 2011: Scales, growth rates, and spectral fluxes of baroclinic instability in the ocean. J. Phys. Oceanogr., 41, 1057-1076, doi:10.1175/2011JPO4404.1.

Uematsu, A., R. Nakamura, Y. Nakajima, and Y. Yajima, 2013: $\mathrm{X}$-band interferometric SAR sensor for the Japanese altimetry mission, COMPIRA. IEEE Int. Geoscience and Remote Sensing Symp. (IGARSS), Melbourne, Victoria, IEEE, 29432946, doi:10.1109/IGARSS.2013.6723442. 